\title{
Large Deviations Performance of Consensus+Inno- vations Distributed Detection With Non-Gaussian Observations
}

\author{
Dragana Bajović, Student Member, IEEE, Dušan Jakovetić, Student Member, IEEE, José M. F. Moura, Fellow, IEEE,
} João Xavier, Member, IEEE, and Bruno Sinopoli, Member, IEEE

\begin{abstract}
We establish the large deviations asymptotic performance (error exponent) of consensus+innovations distributed detection over random networks with generic (non-Gaussian) sensor observations. At each time instant, sensors 1) combine theirs with the decision variables of their neighbors (consensus) and 2) assimilate their new observations (innovations). This paper shows for general non-Gaussian distributions that consensus+innovations distributed detection exhibits a phase transition behavior with respect to the network degree of connectivity. Above a threshold, distributed is as good as centralized, with the same optimal asymptotic detection performance, but, below the threshold, distributed detection is suboptimal with respect to centralized detection. We determine this threshold and quantify the performance loss below threshold. Finally, we show the dependence of the threshold and of the performance on the distribution of the observations: the asymptotic performance of distributed detectors over the same random network with different observations' distributions, for example, Gaussian, Laplace, or quantized, may be different, even though the asymptotic performance of the corresponding centralized detectors is the same.
\end{abstract}

Index Terms-Chernoff information, consensus+innovations, distributed detection, information flow, large deviations, non-Gaussian distributions, performance analysis, random network.

\section{INTRODUCTION}

C ONSIDER a distributed detection scenario where $N$ sensors are connected by a generic network with intermittently failing links. The sensors perform consensus+innovations

Manuscript received November 17, 2011; revised April 03, 2012 and July 07, 2012; accepted July 08, 2012. Date of publication July 31, 2012; date of current version nulldate. The associate editor coordinating the review of this manuscript and approving it for publication was Dr. Ta-Sung Lee. The work of D. Bajović, D. Jakovetić, J. Xavier, and B. Sinopoli was partially supported by the Carnegie-Mellon/Portugal Program managed by ICTI through Grants CMU-PT/SIA/0026/2009, SFRH/BD/33517/2008 and SFRH/BD/33518/2008 and the Fundação para a Ciência e Tecnologia by Grant PTDC/EEA-CRO/104243/2008; and by ISR/IST plurianual funding (POSC program, FEDER). The work of D. Jakovetić and J. M. F. Moura was partially supported by the NSF Grants CCF-1011903 and CCF-1018509, and by the AFOSR Grant FA95501010291. D. Bajović and D. Jakovetić hold fellowships from the Carnegie-Mellon/Portugal Program.

D. Bajović and D. Jakovetić are with the Institute for Systems and Robotics (ISR), Instituto Superior Técnico (IST), Technical University of Lisbon, Lisbon, Portugal. They are also with the Department of Electrical and Computer Engineering, Carnegie Mellon University, Pittsburgh, PA, USA (e-mail: dbajovic@andrew.cmu.edu; djakovet@andrew.cmu.edu; dragana@isr.ist.utl.pt; djakovetic@isr.ist.utl.pt).

J. M. F. Moura and B. Sinopoli are with the Department of Electrical and Computer Engineering, Carnegie Mellon University, Pittsburgh, PA 15213 USA (e-mail: moura@ece.cmu.edu; brunos@ece.cmu.edu).

J. Xavier is with the Institute for Systems and Robotics (ISR), Instituto Superior Técnico (IST), Technical University of Lisbon, Lisbon, Portugal (e-mail: jxavier@isr.ist.utl.pt).

Color versions of one or more of the figures in this paper are available online at http://ieeexplore.ieee.org.

Digital Object Identifier 10.1109/TSP.2012.2210885 distributed detection; in other words, at each time $k$, each sensor $i$ updates its local decision variable $x_{i}(k)$ by: 1$)$ sensing and processing a new measurement to create an intermediate variable; and 2) weight averaging it with its neighbors' intermediate decision variables. We showed in [1] that, when the sensor observations are Gaussian, the consensus+innovations distributed detector exhibits a phase transition. When the network connectivity is above a threshold, then the distributed detector is asymptotically optimal, i.e., asymptotically equivalent to the optimal centralized detector that collects the observations of all sensors.

This paper establishes the asymptotic performance of distributed detection over random networks for generic, non-Gaussian sensor observations. We adopt as asymptotic performance measure the exponential decay rate of the Bayes error probability (error exponent). We show that phase transition behavior emerges with non-Gaussian observations and demonstrate how the optimality threshold is a function of the log-moment generating function of the sensors' observations and of the number of sensors $N$. This reveals a very interesting interplay between the distribution of the sensor observations (e.g., Gaussian or Laplace) and the rate of diffusion (or connectivity) of the network (measured by a parameter $|\log r| \in[0, \infty)$ defined in Section II): for a network with the same connectivity, a distributed detector with say, Laplace observations distributions, may match the optimal asymptotic performance of the centralized detector, while the distributed detector for Gaussian observations may be suboptimal, even though the centralized detectors for the two distributions, Laplace and Gaussian, have the same optimal asymptotic performance.

For distributed detection, we determine the range on the detection threshold $\gamma$ for which each sensor achieves exponentially fast decay of the error probability (strictly positive error exponent), and we find the optimal $\gamma$ that maximizes the error exponent. Interestingly, above the critical (phase transition) value for the network connectivity $|\log r|$, the optimal detector threshold is $\gamma^{\star}=0$, mimicking the (asymptotically) optimal threshold for the centralized detector. However, below the critical connectivity, we show by a numerical example that the optimal distributed detector threshold might be non zero.

Brief review of the literature. A large body of work on distributed detection considers fusion center (FC)-based architectures, e.g., [2]-[7], and, recently, [8], [9]: [8] selects a subset of sensors that optimizes detection performance at the FC; [9] optimizes the local linear precoding of the sensors' messages to the FC, to optimize detection performance subject to a transmit 
power constraint. References [10]-[13] study consensus-based detection. Consensus +innovations estimation is considered by [14]-[18], while different variants of consensus +innovations detection are studied in [19]-[25]. We analyze here running consensus, the variant in [21].

Reference [21] considers asymptotic optimality of running consensus, but in a framework that is very different from ours. Reference [21] studies the asymptotic performance of the distributed detector where the means of the sensor observations under the two hypotheses become closer and closer (vanishing signal to noise ratio (SNR)), at the rate of $\frac{1}{\sqrt{k}}$, where $k$ is the number of observations. For this problem, there is an asymptotic, non-zero, probability of miss and an asymptotic, non-zero, probability of false alarm. Under these conditions, running consensus is as efficient as the optimal centralized detector, [26], as long as the network is connected on average. Here, we assume that the means of the distributions stay fixed as $k$ grows. We establish, through large deviations, the rate (error exponent) at which the error probability decays to zero as $k$ goes to infinity. We show that connectedness on average is not sufficient for running consensus to achieve the optimality of centralized detection; rather, phase transition occurs, with distributed becoming as good as centralized, when the network connectivity, measured by $|\log r|$, exceeds a certain threshold.

We distinguish this paper from our prior work on the performance analysis of running consensus. In [27], we studied deterministically time varying networks and Gaussian observations, and in [28], we considered a different consensus+innovations detector with Gaussian observations and additive communication noise. Here, we consider random networks, nonGaussian observations, and noiseless communications. Reference [1] considers random networks and Gaussian, spatially correlated observations. In contrast, here the observations are non-Gaussian spatially independent. We proved our results in [1] by using the quadratic nature of the Gaussian log-moment generating function. For general non-Gaussian observations, the log-moment generating function is no longer quadratic, and the arguments in [1] no longer apply; we develop a more general methodology that establishes the optimality threshold in terms of the log-moment generating function of the log-likelihood ratio. We derive our results from generic properties of the log-moment generating function like convexity and zero value at the origin. Finally, while [1] and our other prior work considered zero detection threshold $\gamma=0$, here we extend the results to generic detection thresholds $\gamma$. Our analysis reveals that, when $|\log r|$ is above its critical value, the zero detector threshold $\gamma=$ 0 is (asymptotically) optimal. When $|\log r|$ is below the critical value, we compute the optimal detector threshold $\gamma=\gamma^{\star}$, which may be non-zero in general.

Our analysis shows the impact of the distribution of the sensor observations on the performance of distributed detection: distributed detectors (with different distributions of the sensors observations) can have different asymptotic performance, even though the corresponding centralized detectors are equivalent, as we will illustrate in detail in Section IV.

Paper outline. Section II introduces the network and sensor observations models and presents the consensus+innovations distributed detector. Section III presents and proves our main results on the asymptotic performance of the distributed detector.
For a cleaner exposition, this section proves the results for (spatially) identically distributed sensor observations. Section IV illustrates our results on several types of sensor observation distributions, namely, Gaussian, Laplace, and discrete valued distributions, discussing the impact of these distributions on distributed detection performance. Section V extends our main results to non-identically distributed sensors' observations. Finally, Section VI concludes the paper.

Notation. We denote by: $A_{i j}$ the $(i, j)$-th entry of a matrix $A ; a_{i}$ the $i$-th entry of a vector $a ; I, 1$, and $e_{i}$, respectively, the identity matrix, the column vector with unit entries, and the $i$-th column of $I ; J$ the $N \times N$ ideal consensus matrix $J:=\left(\frac{1}{N}\right) 11^{\top}$; $\|\cdot\|_{l}$ the vector (respectively, matrix) $l$-norm of its vector (respectively, matrix) argument; $\|\cdot\|=\|\cdot\|_{2}$ the Euclidean (respectively, spectral) norm of its vector (respectively, matrix) argument; $\mu_{i}(\cdot)$ the $i$-th largest eigenvalue; $\mathbb{E}[\cdot]$ and $\mathbb{P}(\cdot)$ the expected value and probability operators, respectively; $\mathcal{I}_{\mathcal{A}}$ the indicator function of the event $\mathcal{A} ; \nu^{N}$ the product measure of $N$ i.i.d. observations drawn from the distribution with measure $\nu$; $h^{\prime}(z)$ and $h^{\prime \prime}(z)$ the first and the second derivatives of the function $h$ at point $z$.

\section{Problem Formulation}

This section introduces the sensor observations model, reviews the optimal centralized detector, and presents the consensus+innovations distributed detector. The section also reviews relevant properties of the log-moment generating function of a sensor's log-likelihood ratio that are needed in the sequel.

\section{A. Sensor Observations Model}

We study the binary hypothesis testing problem $H_{1}$ versus $H_{0}$. We consider a network of $N$ sensors where $Y_{i}(t)$ is the observation of sensor $i$ at time $t$, and $i=1, \ldots, N, t=1,2, \ldots$

Assumption 1: The sensors' observations $\left\{Y_{i}(t)\right\}$ are independent and identically distributed (i.i.d.) both in time and in space, with distribution $\nu_{1}$ under hypothesis $H_{1}$ and $\nu_{0}$ under $H_{0}$ :

$$
Y_{i}(t) \sim\left\{\begin{array}{ll}
\nu_{1}, & H_{1} \\
\nu_{0}, & H_{0}
\end{array}, i=1, \ldots, N, t=1,2, \ldots\right.
$$

Here $\nu_{1}$ and $\nu_{0}$ are mutually absolutely continuous, distinguishable measures. The prior probabilities $\pi_{1}=\mathbb{P}\left(H_{1}\right)$ and $\pi_{0}=$ $\mathbb{P}\left(H_{0}\right)=1-\pi_{1}$ are in $(0,1)$.

By spatial independence, the joint distribution of the observations of all sensors

$$
Y(t):=\left(Y_{1}(t), \ldots, Y_{N}(t)\right)^{\top}
$$

at any time $t$ is $\nu_{1}^{N}$ under $H_{1}$ and $\nu_{0}^{N}$ under $H_{0}$. Our main results in Section III are derived under Assumption 1. Section V extends them to non-identical (but still independent) sensors' observations.

\section{B. Centralized Detection, Log-Moment Generating Function (LMGF), and Optimal Error Exponent}

The log-likelihood ratio of sensor $i$ at time $t$ is $L_{i}(t)$ and given by

$$
L_{i}(t)=\log \frac{f_{1}\left(Y_{i}(t)\right)}{f_{0}\left(Y_{i}(t)\right)},
$$


where, $f_{l}(\cdot), l=0,1$, is 1$)$ the probability density function corresponding to $\nu_{l}$, when $Y_{i}(t)$ is an absolutely continuous random variable; or 2) the probability mass function corresponding to $\nu_{l}$, when $Y_{i}(t)$ is discrete valued.

Under Assumption 1, the log-likelihood ratio test for $k$ time observations from all sensors, for a threshold $\gamma$ is: ${ }^{1}$

$$
D(k):=\frac{1}{N k} \sum_{t=1}^{k} \sum_{i=1}^{N} L_{i}(t) \underset{H_{1}}{\stackrel{H_{0}}{\gtrless}} \gamma .
$$

Log-moment generating function (LMGF). We introduce the LMGF of $L_{i}(t)$ and its properties that play a major role in assessing the performance of distributed detection. Let $\Lambda_{l}(l=$ $0,1)$ denote the LMGF for the log-likelihood ratio under hypothesis $H_{l}$ :

$$
\Lambda_{l}: \mathbb{R} \longrightarrow(-\infty,+\infty], \quad \Lambda_{l}(\lambda)=\log \mathbb{E}\left[e^{\lambda L_{1}(1)} \mid H_{l}\right] .
$$

In (4), $L_{1}(1)$ replaces $L_{i}(t)$, for arbitrary $i=1, \ldots, N$, and $t=$ $1,2, \ldots$, due to the spatial and temporal identically distributed observations, see Assumption 1.

Lemma 1: Consider Assumption 1. For $\Lambda_{0}$ and $\Lambda_{1}$ in (4) the following holds:

(a) $\Lambda_{0}$ is convex;

(b) $\Lambda_{0}(\lambda) \in(-\infty, 0)$, for $\lambda \in(0,1), \Lambda_{0}(0)=\Lambda_{0}(1)=0$, and $\Lambda_{l}^{\prime}(0)=\mathbb{E}\left[L_{1}(1) \mid H_{l}\right], l=0,1$

(c) $\Lambda_{1}(\lambda)$ satisfies:

$$
\Lambda_{1}(\lambda)=\Lambda_{0}(\lambda+1), \quad \text { for } \lambda \in \mathbb{R} .
$$

Proof: For a proof of (a) and (b), see [29]. Part (c) follows from the definitions of $\Lambda_{0}$ and $\Lambda_{1}$, which we show here for the case when the distributions $\nu_{1}$ and $\nu_{0}$ are absolutely continuous (the proof for discrete distributions is similar):

$$
\begin{aligned}
\Lambda_{1}(\lambda) & =\log \mathbb{E}\left[e^{\lambda L_{1}(1)} \mid H_{1}\right] \\
& =\log \int_{y \in \mathbb{R}}\left(\frac{f_{1}(y)}{f_{0}(y)}\right)^{\lambda} f_{1}(y) d y \\
& =\log \int_{y \in \mathbb{R}}\left(\frac{f_{1}(y)}{f_{0}(y)}\right)^{1+\lambda} f_{0}(y) d y \\
& =\Lambda_{0}(1+\lambda) .
\end{aligned}
$$

We further assume that the LMGF of a sensor's observation is finite.

Assumption 2: $\quad \Lambda_{0}(\lambda)<+\infty, \forall \lambda \in \mathbb{R}$.

In the next two remarks, we give two classes of problems when Assumption 2 holds.

Remark I: We consider the signal+noise model:

$$
Y_{i}(t)= \begin{cases}m+n_{i}(t), & H_{1} \\ n_{i}(t), & H_{0}\end{cases}
$$

Here $m \neq 0$ is a constant signal and $n_{i}(t)$ is a zero-mean additive noise with density function $f_{n}(\cdot)$ supported on $\mathbb{R}$; we rewrite $f_{n}(\cdot)$, without loss of generality, as $f_{n}(y)=c e^{-g(y)}$,

\footnotetext{
${ }^{1}$ In (3), we re-scale the spatio-temporal sum of the log-likelihood ratios $L_{i}(t)$ by dividing the sum by $N k$. Note that we can do so without loss of generality, as the alternative test without re-scaling is: $\sum_{t=1}^{k} \sum_{i=1}^{N} L_{i}(t) \underset{H_{1}}{\stackrel{H_{0}}{\gtrless}} \gamma^{\prime}$, with $\gamma^{\prime}=N k \gamma$.
}

where $c>0$ is a constant. Then, the Appendix shows that Assumption 2 holds under the following mild technical condition: either one of (7) or (8) and one of (9) or (10) hold:

$$
\lim _{y \rightarrow+\infty} \frac{g(y)}{|y|^{\tau_{+}}}=\rho_{+}, \text {for some } \rho_{+}, \tau_{+} \in(0,+\infty)
$$

$$
\begin{aligned}
& \lim _{y \rightarrow+\infty} \frac{g(y)}{(\log (|y|))^{\mu_{+}}}=\rho_{+}, \text {for some } \rho_{+} \in(0,+\infty), \\
& \mu_{+} \in(1,+\infty) \\
& \lim _{y \rightarrow-\infty} \frac{g(y)}{|y|^{\tau_{-}}}=\rho_{-}, \text {for some } \rho_{-}, \tau_{-} \in(0,+\infty)
\end{aligned}
$$

$$
\begin{gathered}
\lim _{y \rightarrow-\infty} \frac{g(y)}{(\log (|y|))^{\mu_{-}}}=\rho_{-}, \text {for some } \rho_{-} \in(0,+\infty), \\
\mu_{-} \in(1,+\infty) .
\end{gathered}
$$

In (8) and (10), we can also allow either (or both) $\mu_{+}, \mu_{-}$to equal 1 , but then the corresponding $\rho$ is in $(1, \infty)$. Note that $f_{n}(\cdot)$ need not be symmetric, i.e., $f_{n}(y)$ need not be equal to $f_{n}(-y)$. Intuitively, the tail of the density $f_{n}(\cdot)$ behaves regularly, and $g(y)$ grows either like a polynomial of arbitrary finite order in $y$, or slower, like a power $y^{\tau}, \tau \in(0,1)$, or like a $\operatorname{logarithm} c(\log y)^{\mu}$. The class of admissible densities $f_{n}(\cdot)$ includes power laws $c y^{-p}, p>1$, or the exponential families $e^{\theta \phi(y)-A(\theta)}, A(\theta):=\log \int_{y=-\infty}^{+\infty} e^{\theta \phi(y)} \chi(d y)$, with: 1) the Lebesgue base measure $\chi ; 2)$ the polynomial, power, or logarithmic potentials $\phi(\cdot)$; and 3 ) the canonical set of parameters $\theta \in \Theta=\{\theta: A(\theta)<+\infty\}$, [30].

Remark II: Assumption 2 is satisfied if $Y_{i}(k)$ has arbitrary (different) distributions under $H_{1}$ and $H_{0}$ with the same, compact support; a special case is when $Y_{i}(k)$ is discrete, supported on a finite alphabet.

Centralized detection: Asymptotic performance. We consider briefly the performance of the centralized detector that will benchmark the performance of the distributed detector. Denote by $\gamma_{l}:=\mathbb{E}\left[L_{1}(1) \mid H_{l}\right], l=0,1$. It can be shown [31] that $\gamma_{0}<0$ and $\gamma_{1}>0$. Now, consider the centralized detector in (3) with the constant thresholds $\gamma$, for all $k$, and denote by:

$$
\begin{aligned}
\alpha(k, \gamma) & =\mathbb{P}\left(D(k) \geq \gamma \mid H_{0}\right), \\
\beta(k, \gamma) & =\mathbb{P}\left(D(k)<\gamma \mid H_{1}\right), \\
P_{\mathrm{e}}(k, \gamma) & =\alpha(k, \gamma) \pi_{0}+\beta(k, \gamma) \pi_{1},
\end{aligned}
$$

respectively, the probability of false alarm, probability of miss, and Bayes (average) error probability. In this paper, we adopt the minimum Bayes error probability criterion, both for the centralized and later for our distributed detector, and, from now on, we refer to it simply as the error probability. A standard Theorem (Theorem 3.4.3., [31]) says that, for any choice of $\gamma \in$ $\left(\gamma_{0}, \gamma_{1}\right)$, the error probability decays exponentially fast to zero in $k$. For $\gamma \notin\left(\gamma_{0}, \gamma_{1}\right)$, the error probability does not converge to zero at all. To see this, assume that $H_{1}$ is true, and let $\gamma \geq \gamma_{1}$. Then, by noting that $\mathbb{E}\left[D(k) \mid H_{1}\right]=\gamma_{1}$, for all $k$, we have that $\beta(k, \gamma)=\mathbb{P}\left(D(k)<\gamma \mid H_{1}\right) \geq \mathbb{P}\left(D(k) \leq \gamma_{1} \mid H_{1}\right) \rightarrow \frac{1}{2}$ as $k \rightarrow \infty$, by the central limit theorem.

Denote by $I_{l}(\cdot), l=0,1$, the Fenchel-Legendre transform [31] of $\Lambda_{l}(\cdot)$ :

$$
I_{l}(z)=\sup _{\lambda \in \mathbb{R}} \lambda z-\Lambda_{l}(\lambda), z \in \mathbb{R} .
$$


It can be shown [31] that $I_{l}(\cdot)$ is nonnegative, strictly convex (unless $L_{1}(1)$ is an almost sure constant), $I_{l}\left(\gamma_{l}\right)=0$, for $l=$ 0,1 , and $I_{1}(z)=I_{0}(z)-z$, [31]. We now state the result on the centralized detector's asymptotic performance.

Lemma 2: Let Assumption 1 hold, and consider the family of centralized detectors (3) with the constant threshold $\gamma \in$ $\left(\gamma_{0}, \gamma_{1}\right)$. Then, the best (maximal) error exponent:

$$
\lim _{k \rightarrow \infty}-\frac{1}{k} \log P_{\mathrm{e}}(k, \gamma)
$$

is achieved for the zero threshold $\gamma=0$ and equals $N C_{\text {ind }}$, where $C_{\text {ind }}=I_{0}(0)$.

The quantity $C_{\text {ind }}$ is referred to as the Chernoff information of a single sensor observation $Y_{i}(t)$. Lemma 2 says that the centralized detector' error exponent is $N$ times larger than an individual sensor's error exponent. We remark that, even if we allow for time-varying thresholds $\gamma_{k}=\gamma$, the error exponent $N C_{\text {ind }}$ cannot be improved, i.e., the centralized detector with zero threshold is asymptotically optimal over all detectors. We will see that, when a certain condition on the network connectivity holds, the distributed detector is asymptotically optimal, i.e., achieves the best error exponent $N C_{\text {ind }}$, and the zero threshold is again optimal. However, when the network connectivity condition is not met, the distributed detector is no longer asymptotically optimal, and the optimal threshold may be non zero.

Proof of Lemma 2: Denote by $\Lambda_{0, N}$ the LMGF for the log-likelihood ratio $\sum_{i=1}^{N} L_{i}(t)$ for the observations of all sensors at time $t$. Then, $\Lambda_{0, N}(\lambda)=N \Lambda_{0}(\lambda)$, by the i.i.d. in space assumption on the sensors' observations. The Lemma now follows by the Chernoff lemma (Corollary 3.4.6, [31]):

$$
\begin{aligned}
\lim _{k \rightarrow \infty}-\frac{1}{k} \log P_{\mathrm{e}}(k, 0) & =\max _{\lambda \in[0,1]}\left\{-\Lambda_{0, N}(\lambda)\right\} \\
& =N \max _{\lambda \in[0,1]}\left\{-\Lambda_{0}(\lambda)\right\}=N I_{0}(0) .
\end{aligned}
$$

\section{Distributed Detection Algorithm}

We now consider distributed detection when the sensors cooperate through a randomly varying network. Specifically, we consider the running consensus distributed detector proposed in [21]. Each sensor $i$ maintains its local decision variable $x_{i}(k)$, which is a local estimate of the global optimal decision variable $D(k)$ in (3). Note that $D(k)$ is not locally available. At each time $k$, each sensor $i$ updates $x_{i}(k)$ in two ways: 1) by incorporating its new observation $Y_{i}(k)$ to make an intermediate decision variable $\frac{k-1}{k} x_{i}(k-1)+\frac{1}{k} L_{i}(k)$; and 2) by exchanging the intermediate decision variable locally with its neighbors and computing the weighted average of its own and the neighbors' intermediate variables.

More precisely, the update of $x_{i}(k)$ is as follows:

$$
\begin{array}{r}
x_{i}(k)=\sum_{j \in O_{i}(k)} W_{i j}(k)\left(\frac{k-1}{k} x_{j}(k-1)+\frac{1}{k} L_{j}(k)\right), \\
k=1,2, \ldots x_{i}(0)=0 .
\end{array}
$$

Here $O_{i}(k)$ is the (random) neighborhood of sensor $i$ at time $k$ (including $i$ ), and $W_{i j}(k)$ are the (random) averaging weights. The sensor $i$ 's local decision test at time $k$ is:

$$
x_{i}(k) \underset{H_{1}}{\stackrel{H_{0}}{\gtrless}} \gamma,
$$

i.e., $H_{1}$ (respectively, $H_{0}$ ) is decided when $x_{i}(k) \geq \gamma($ respectively, $\left.x_{i}(k)<\gamma\right)$.

Write the consensus+innovations algorithm (13) in vector form. Let $x(k)=\left(x_{1}(k), x_{2}(k), \ldots, x_{N}(k)\right)^{\top}$ and $L(k)=\left(L_{1}(k), \ldots, L_{N}(k)\right)^{\top}$. Also, collect the averaging weights $W_{i j}(k)$ in the $N \times N$ matrix $W(k)$, where, clearly, $W_{i j}(k)=0$ if the sensors $i$ and $j$ do not communicate at time step $k$. The algorithm (13) becomes:

$$
\begin{aligned}
x(k)=W(k)\left(\frac{k-1}{k} x(k-1)+\frac{1}{k} L(k)\right), & \\
k & =1,2, \ldots x_{i}(0)=0 .
\end{aligned}
$$

Network model. We state the assumption on the random averaging matrices $W(k)$.

Assumptions 3: The averaging matrices $W(k)$ satisfy the following:

(a) The sequence $\{W(k)\}_{k=1}^{\infty}$ is i.i.d.

(b) $W(k)$ is symmetric and stochastic (row-sums equal 1 and $W_{i j}(k) \geq 0$ ) with probability one, $\forall k$.

(c) There exists $\eta>0$, such that, for any realization $W(k)$, $W_{i i}(k) \geq \eta, \forall i$, and, $W_{i j}(k) \geq \eta$ whenever $W_{i j}(k)>0$, $i \neq j$

(d) $W(k)$ and $Y(t)$ are mutually independent over all $k$ and $t$.

Condition (c) is mild and says that: 1) sensor $i$ assigns a nonnegligible weight to itself; and 2) when sensor $i$ receives a message from sensor $j$, sensor $i$ assigns a non-negligible weight to sensor $j$.

Define the matrices $\Phi(k, t)$ by:

$$
\Phi(k, t):=W(k) W(k-1) \ldots W(t), k \geq t \geq 1 .
$$

It is easy to verify from $(15)$ that $x(k)$ equals:

$$
x(k)=\frac{1}{k} \sum_{t=1}^{k} \Phi(k, t) L(t), k=1,2, \ldots .
$$

Choice of threshold $\gamma$. We restrict the choice of threshold $\gamma$ to $\gamma \in\left(\gamma_{0}, \gamma_{1}\right), \gamma_{0}<0, \gamma_{1}>0$, where we recall $\gamma_{l}=$ $\mathbb{E}\left[L_{1}(1) \mid H_{l}\right], l=0,1$. Namely, $W(t)$ is a stochastic matrix, hence $W(t) 1=1$, for all $t$, and thus $\Phi(k, t) 1=1$. Also, $\mathbb{E}\left[L(t) \mid H_{l}\right]=\gamma_{l} 1$, for all $t, l=0,1$. Now, by iterating expectation:

$$
\begin{aligned}
\mathbb{E}\left[x(k) \mid H_{l}\right] & =\mathbb{E}\left[\mathbb{E}\left[x(k) \mid H_{l}, W(1), \ldots, W(k)\right]\right] \\
& =\mathbb{E}\left[\frac{1}{k} \sum_{t=1}^{k} \Phi(k, t) \mathbb{E}\left[L(t) \mid H_{l}\right]\right] \\
& =\gamma_{l} 1, l=0,1,
\end{aligned}
$$

and so $\mathbb{E}\left[x_{i}(k) \mid H_{l}\right]=\gamma_{l}$, for all $i, k$. Moreover, it can be shown (proof is omitted due to lack of space) that $x_{i}(k)$ converges in probability to $\gamma_{l}$ under $H_{l}$. Now, a similar argument as with the centralized detector in Section II-B shows that for $\gamma \notin\left(\gamma_{0}, \gamma_{1}\right)$, 
the error probability with detector (13) and (14) does not converge to zero when $k \rightarrow \infty$. We will show that, for any $\gamma \in$ $\left(\gamma_{0}, \gamma_{1}\right)$, the error probability converges to 0 exponentially fast, and we find the optimal $\gamma=\gamma^{\star}$ that maximizes a certain lower bound on the exponent of the error probability.

Network connectivity. From (17), we can see that the matrices $\Phi(k, t)$ should be as close to $J$ as possible for enhanced detection performance. Namely, the ideal (unrealistic) case when $\Phi(k, t) \equiv J$ for all $k, t$, corresponds to the scenario where each sensor $i$ is equivalent to the optimal centralized detector. It is well known that, under certain conditions, the matrices $\Phi(k, t)$ converge in probability to $J$ :

$$
\mathbb{P}(\|\Phi(k, t)-J\|>\epsilon) \rightarrow 0 \text { as }(k-t) \rightarrow \infty, \epsilon \in(0,1),
$$

such that $\mathbb{P}(\|\Phi(k, t)-J\|>\epsilon)$ vanishes exponentially fast in $(k-t)$, i.e., $\mathbb{P}(\|\Phi(k, t)-J\|>\epsilon) \approx r^{(k-t)}, r \in[0,1]$. The quantity $r$ determines the speed of convergence of the matrices $\Phi(k, t)$. The closer to zero $r$ is, the faster consensus is. We refer to $|\log r|$ as the network connectivity. We will see that the distributed detection performance significantly depends on $r$. Formally, $|\log r|=-\log r$ is given by: 2

$$
|\log r|:=\lim _{(k-t) \rightarrow \infty}-\frac{1}{k-t} \log \mathbb{P}(\|\Phi(k, t)-J\|>\epsilon) .
$$

For the exact calculation of $r$, we refer to [32]. Reference [32] shows that, for the commonly used models of $W(k)$, gossip and link failure (links in the underlying network fail independently, with possibly mutually different probabilities), $r$ is easily computable, by solving a certain min-cut problem. In general, $r$ is not easily computable, but all our results (Theorem 5, Corollary 6 , Corollary 11) hold when $r$ is replaced by an upper bound. An upper bound on $r$ is given by $\mu_{2}\left(\mathbb{E}\left[W^{2}(k)\right]\right)$, [32].

The following Lemma easily follows from (18).

Lemma 4: Let Assumption 3 hold. Then, for any $\delta \in(0,|\log r|)$, there exists a constant $C(\delta) \in(0, \infty)$ (independent of $\epsilon \in(0,1))$ such that, for any $\epsilon \in(0,1)$ :

$$
\begin{array}{r}
\mathbb{P}(\|\Phi(k, t)-J\|>\epsilon) \\
\leq C(\delta) e^{-(k-t)(|\log r|-\delta)}, \text { for all } k \geq t .
\end{array}
$$

\section{MAIN RESUlts: AsYMPtOTIC ANALYSIS AND ERROR EXPONENTS FOR DISTRIBUTED DETECTION}

Section III-A establishes the asymptotic performance of consensus+innovations distributed detection under identically distributed sensors' observations; Section III-B proves the results.

\section{A. Statement of Main Results}

In this section, we analyze the performance of distributed detection in terms of the detection error exponent, when the number of observations (per sensor), or the size $k$ of the observation interval tends to $+\infty$. In particular, we show that there exists a threshold on the network connectivity $|\log r|$ such that if $|\log r|$ is above this threshold, each sensor in the network achieves asymptotic optimality (i.e., the error exponent at each sensor is the total Chernoff information equal to $N C_{\text {ind }}$ ). When $|\log r|$ is below the threshold, we give a lower bound for the error exponent. Both the threshold and the lower bound are

\footnotetext{
${ }^{2}$ It can be shown that the limit in (18) exists and that it does not depend on $\epsilon$
} for $\epsilon \in(0,1)$. given solely in terms of the log-moment generating function $\Lambda_{0}$ and the number of sensors $N$. These findings are summarized in Theorem 5 and Corollary 6 below.

Let $\alpha_{i}(k, \gamma), \beta_{i}(k, \gamma)$, and $P_{\mathrm{e}, \mathrm{i}}(k, \gamma)$ denote the probability of false alarm, the probability of miss, and the error probability, respectively, of sensor $i$ for the detector (13) and (14), for the threshold equal to $\gamma$ :

$$
\begin{aligned}
\alpha_{i}(k, \gamma) & =\mathbb{P}\left(x_{i}(k) \geq \gamma \mid H_{0}\right), \\
\beta_{i}(k, \gamma) & =\mathbb{P}\left(x_{i}(k)<\gamma \mid H_{1}\right), \\
P_{\mathrm{e}, \mathrm{i}}(k, \gamma) & =\pi_{0} \alpha_{i}(k ; \gamma)+\pi_{1} \beta_{i}(k ; \gamma),
\end{aligned}
$$

where, we recall, $\pi_{1}$ and $\pi_{0}$ are the prior probabilities. Also, recall $I_{l}(\cdot), l=0,1$, in (12).

Theorem 5: Let Assumptions 1-3 hold and consider the family of distributed detectors in (13) and (14) parameterized by detection thresholds $\gamma \in\left(\gamma_{0}, \gamma_{1}\right)$. Let $\lambda_{l}^{\mathrm{s}}$ be the zero of the function:

$$
\Delta_{l}(\lambda):=\Lambda_{l}(N \lambda)-|\log r|-N \Lambda_{l}(\lambda), l=0,1,
$$

and define $\gamma_{l}^{-}, \gamma_{l}^{+}, l=0,1$ by

$$
\begin{aligned}
& \gamma_{0}^{-}=\Lambda_{0}^{\prime}\left(\lambda_{0}^{\mathrm{s}}\right), \gamma_{0}^{+}=\Lambda_{0}^{\prime}\left(N \lambda_{0}^{\mathrm{s}}\right) \geq \gamma_{0}^{-} \\
& \gamma_{1}^{-}=\Lambda_{1}^{\prime}\left(N \lambda_{1}^{\mathrm{s}}\right), \gamma_{1}^{+}=\Lambda_{1}^{\prime}\left(\lambda_{1}^{\mathrm{s}}\right) \geq \gamma_{1}^{-} .
\end{aligned}
$$

Then, for every $\gamma \in\left(\gamma_{0}, \gamma_{1}\right)$, at each sensor $i, i=1, \ldots, N$, we have:

$$
\begin{aligned}
& \liminf _{k \rightarrow \infty}-\frac{1}{k} \log \alpha_{i}(k, \gamma) \geq B_{0}(\gamma), \\
& \liminf _{k \rightarrow \infty}-\frac{1}{k} \log \beta_{i}(k, \gamma) \geq B_{1}(\gamma),
\end{aligned}
$$

where

$$
\begin{aligned}
B_{0}(\gamma) & =\max _{\lambda \in[0,1]}\left\{N \gamma \lambda-\max \left\{N \Lambda_{0}(\lambda), \Lambda_{0}(N \lambda)-|\log r|\right\}\right\} \\
& = \begin{cases}N I_{0}(\gamma), & \gamma \in\left(\gamma_{0}, \gamma_{0}^{-}\right] \\
N I_{0}\left(\gamma_{0}^{-}\right)+N \lambda_{0}^{\mathrm{s}}\left(\gamma-\gamma_{0}^{-}\right), & \gamma \in\left(\gamma_{0}^{-}, \gamma_{0}^{+}\right) \\
I_{0}(\gamma)+|\log r|, & \gamma \in\left[\gamma_{0}^{+}, \gamma_{1}\right)\end{cases} \\
B_{1}(\gamma) & =\max _{\lambda \in[-1,0]}\left\{N \gamma \lambda-\max \left\{N \Lambda_{1}(\lambda), \Lambda_{1}(N \lambda)-|\log r|\right\}\right\} \\
& = \begin{cases}I_{1}(\gamma)+|\log r|, & \gamma \in\left(\gamma_{0}, \gamma_{1}^{-}\right] \\
N I_{1}\left(\gamma_{1}^{+}\right)+N \lambda_{1}^{\mathrm{s}}\left(\gamma-\gamma_{1}^{+}\right), & \gamma \in\left(\gamma_{1}^{-}, \gamma_{1}^{+}\right) \\
N I_{1}(\gamma), & \gamma \in\left[\gamma_{1}^{+}, \gamma_{1}\right) .\end{cases}
\end{aligned}
$$

Corollary 6: Let Assumptions 1-3 hold and consider the family of distributed detectors in (13) and (14) parameterized by the detector threshold $\gamma \in\left(\gamma_{0}, \gamma_{1}\right)$. Then:

(a)

$$
\liminf _{k \rightarrow \infty}-\frac{1}{k} \log P_{\mathrm{e}, \mathrm{i}}(k, \gamma) \geq \min \left\{B_{0}(\gamma), B_{1}(\gamma)\right\}>0,
$$

and the lower bound in (24) is maximized for the point $\gamma^{\star} \in\left(\gamma_{0}, \gamma_{1}\right)^{3}$ at which $B_{0}\left(\gamma^{\star}\right)=B_{1}\left(\gamma^{\star}\right)$.

(b) Consider $\lambda^{\bullet}=\arg \min _{\lambda \in \mathbb{R}} \Lambda_{0}(\lambda)$, and let:

$$
\begin{aligned}
\operatorname{thr}\left(\Lambda_{0}, N\right)=\max & \left\{\Lambda_{0}\left(N \lambda^{\bullet}\right)-N \Lambda_{0}\left(\lambda^{\bullet}\right),\right. \\
& \left.\Lambda_{0}\left(1-N\left(1-\lambda^{\bullet}\right)\right)-N \Lambda_{0}\left(\lambda^{\bullet}\right)\right\} .
\end{aligned}
$$

\footnotetext{
${ }^{3}$ As we show in the proof, such a point exists and is unique.
} 



Fig. 1. Top: Illustration of the error exponent lower bounds $B_{0}(\gamma)$ and $B_{1}(\gamma)$ in Theorem 5; Bottom: Illustration of the function $\Phi_{0}(\lambda)$ in (37), and the quantities in (21). We consider $N=3$ sensors and a discrete distribution of $Y_{i}(t)$ over a 5-point alphabet, with the distribution $[.2, .2, .2, .2, .2]$ under $H_{1}$, and $[0.01,0.01,0.01,0.01,0.96]$ under $H_{0}$. We set here $r=0.4$.

Then, when $|\log r| \geq \operatorname{thr}\left(\Lambda_{0}, N\right)$, each sensor $i$ with the detector threshold set to $\gamma=0$, is asymptotically optimal:

$$
\lim _{k \rightarrow \infty}-\frac{1}{k} \log P_{\mathrm{e}, \mathrm{i}}(k, 0)=N C_{\text {ind }}
$$

(c) Suppose $\Lambda_{0}(\lambda)=\Lambda_{0}(1-\lambda)$, for $\lambda \in[0,1]$. Then, $\gamma^{\star}=$ 0 , irrespective of the value of $r$ (even when $|\log r|<$ $\operatorname{thr}\left(\Lambda_{0}, N\right)$.)

Fig. 1 (top) illustrates the error exponent lower bounds $B_{0}(\gamma)$ and $B_{1}(\gamma)$ in Theorem 5, while Fig. 1 (bottom) illustrates the quantities in (21). (See the definition of the function $\Phi_{0}(\lambda)$ in (37) in the proof of Theorem 5.) Note that $B_{l}(\cdot)$ is the convex envelope of the functions $N I_{l}(\cdot)$ and $I_{0}(\cdot)+|\log r|, l=0,1$. We consider $N=3$ sensors and a discrete distribution of $Y_{i}(t)$ over a 5-point alphabet, with the distribution $[.2, .2, .2, .2, .2]$ under $H_{1}$, and $[0.01,0.01,0.01,0.01,0.96]$ under $H_{0}$. We set here $r=0.4$.

Remark: Consider part (c) of Corollary 6. When $\Lambda_{0}(\lambda)=$ $\Lambda_{0}(1-\lambda)$, for $\lambda \in[0,1]$, it can be shown that $\gamma_{0}=-\gamma_{1}<0$, and $B_{0}(\gamma)=B_{1}(-\gamma)$, for all $\gamma \in\left(\gamma_{0}, \gamma_{1}\right)$. This implies that the point $\gamma^{\star}$ at which $B_{0}$ and $B_{1}$ are equal is necessarily zero, and hence the optimal detector threshold $\gamma^{\star}=0$, irrespective of the network connectivity $|\log r|$ (even when $|\log r|<$ $\operatorname{thr}\left(\Lambda_{0}, N\right)$.) This symmetry holds for the Gaussian and Laplace distribution considered in Section IV.

Corollary 6 states that, when the network connectivity $|\log r|$ is above a threshold, the distributed detector in (13) and (14) is asymptotically equivalent to the optimal centralized detector. The corresponding optimal detector threshold is $\gamma=0$. When $|\log r|$ is below the threshold, Corollary 6 determines what value of the error exponent the distributed detector can achieve, for any given $\gamma \in\left(\gamma_{0}, \gamma_{1}\right)$. Moreover, Corollary 6 finds the optimal detector threshold $\gamma^{\star}$ for a given $r ; \gamma^{\star}$ can be found as the unique zero of the strictly decreasing function $\Delta_{B}(\gamma):=B_{1}(\gamma)-B_{0}(\gamma)$ on $\gamma \in\left(\gamma_{0}, \gamma_{1}\right)$, see the proof of Corollary 6 by bisection on $\left(\gamma_{0}, \gamma_{1}\right)$.

Corollary 6 establishes that there exists a "sufficient" connectivity, say $\left|\log r^{\star}\right|$, so that further improvement on the connectivity (and further spending of resources, e.g., transmission power) does not lead to a pay off in terms of detection performance. Hence, Corollary 6 is valuable in the practical design of a sensor network, as it says how much connectivity (resources) is sufficient to achieve asymptotically optimal detection.

Equation (24) says that the distribution of the sensor observations (through LMGF) plays a role in determining the performance of distributed detection. We illustrate and explain by examples this effect in Section IV.

\section{Proofs of the Main Results}

We first prove Theorem 5.

Proof of Theorem 5: Consider the probability of false alarm $\alpha_{i}(k, \gamma)$ in (19). We upper bound $\alpha_{i}(k, \gamma)$ using the exponential Markov inequality [33] parameterized by $\zeta \geq 0$ :

$$
\begin{aligned}
\alpha_{i}(k, \gamma) & =\mathbb{P}\left(x_{i}(k) \geq \gamma \mid H_{0}\right) \\
& =\mathbb{P}\left(e^{\zeta x_{i}(k)} \geq e^{\zeta \gamma} \mid H_{0}\right) \\
& \leq \mathbb{E}\left[e^{\zeta x_{i}(k)} \mid H_{0}\right] e^{-\zeta \gamma} .
\end{aligned}
$$

Next, by setting $\zeta=N k \lambda$, with $\lambda \geq 0$, we obtain:

$$
\begin{aligned}
\alpha_{i}(k, \gamma) & \leq \mathbb{E}\left[e^{N k \lambda x_{i}(k)} \mid H_{0}\right] e^{-N k \lambda \gamma} \\
& =\mathbb{E}\left[e^{N \lambda \sum_{t=1}^{k} \sum_{j=1}^{N} \Phi_{i, j}(k, t) L_{j}(t)} \mid H_{0}\right] e^{-N k \lambda \gamma}
\end{aligned}
$$

The terms in the sum in the exponent in (28) are conditionally independent, given the realizations of the averaging matrices $W(t), t=1, \ldots, k$. Thus, by iterating the expectations, and using the definition of $\Lambda_{0}$ in (4), we compute the expectation in (28) by conditioning first on $W(t), t=1, \ldots, k$ :

$$
\begin{gathered}
\mathbb{E}\left[e^{N \lambda \sum_{t=1}^{k} \sum_{j=1}^{N} \Phi_{i, j}(k, t) L_{j}(t)} \mid H_{0}\right] \\
=\mathbb{E}\left[\mathbb { E } \left[e^{N \lambda \sum_{t=1}^{k} \sum_{j=1}^{N} \Phi_{i, j}(k, t) L_{j}(t)} \mid\right.\right. \\
\left.\left.H_{0}, W(1), \ldots, W(k)\right]\right] \\
=\mathbb{E}\left[e^{\sum_{t=1}^{k} \sum_{j=1}^{N} \Lambda_{0}\left(N \lambda \Phi_{i, j}(k, t)\right)}\right] .
\end{gathered}
$$

Partition of the sample space. We handle the random matrix realizations $W(t), t=1, \ldots, k$, through a suitable partition of the underlying probability space. Adapting an argument from [1], partition the probability space based on the time of the last successful averaging. In more detail, for a fixed $k$, introduce 
the partition $\mathcal{P}_{k}$ of the sample space that consists of the disjoint events $\mathcal{A}_{s, k}, s=0,1, \ldots, k$, given by:

$$
\mathcal{A}_{s, k}=\{\|\Phi(k, s)-J\| \leq \epsilon \text { and }\|\Phi(k, s+1)-J\|>\epsilon\},
$$

for $s=1, \ldots, k-1, \mathcal{A}_{0, k}=\{\|\Phi(k, 1)-J\|>\epsilon\}$, and $A_{k, k}=\{\|\Phi(k, k)-J\| \leq \epsilon\}$. For simplicity of notation, we drop the index $k$ in the sequel and denote event $\mathcal{A}_{s, k}$ by $\mathcal{A}_{s}$, $s=0, \ldots, k$ for $\epsilon>0$. Intuitively, the smaller $t$ is, the closer the product $\Phi(k, t)$ to $J$ is; if the event $\mathcal{A}_{s}$ occurrs, then the largest $t$ for which the product $\Phi(k, t)$ is still $\epsilon$-close to $J$ equals $s$. We now show that $\mathcal{P}_{k}$ is indeed a partition. We need the following simple Lemma. The Lemma shows that convergence of $\Phi(k, s)-J$ is monotonic, for any realization of the matrices $W(1), W(2), \ldots, W(k)$.

Lemma 7: Let Assumption 3 hold. Then, for any realization of the matrices $W(1), \ldots, W(k)$ :

$\|\Phi(k, s)-J\| \leq\|\Phi(k, t)-J\|$, for $1 \leq s \leq t \leq k$.

Proof: Since every realization of $W(t)$ is stochastic and symmetric for every $t$, we have that $W(t) 1=1$ and $1^{\top} W(t)=$ $1^{\top}$, and, so: $\Phi(k, s)-J=W(k) \cdots W(s)-J=(W(k)-$ $J) \cdots(W(s)-J)$. Now, using the sub-multiplicative property of the spectral norm, we get

$$
\begin{aligned}
& \|\Phi(k, s)-J\| \\
& =\|(W(k)-J) \cdots(W(t)-J) \\
& \quad \times(W(t-1)-J) \cdots(W(s)-J) \| \\
& \leq\|(W(k)-J) \cdots(W(t)-J)\| \\
& \quad \times\|(W(t-1)-J)\| \cdots\|(W(s)-J)\| .
\end{aligned}
$$

To prove Lemma 7, it remains to show that $\|W(t)-J\| \leq 1$, for any realization of $W(t)$. To this end, fix a realization $W$ of $W(t)$. Consider the eigenvalue decomposition $W=Q M Q^{\top}$, where $M=\operatorname{diag}\left(\mu_{1}, \ldots, \mu_{N}\right)$ is the matrix of eigenvalues of $W$, and the columns of $Q$ are the orthonormal eigenvectors. As $\frac{1}{\sqrt{N}} 1$ is the eigenvector associated with eigenvalue $\mu_{1}=1$, we have that $W-J=Q M^{\prime} Q^{\top}$, where $M=\operatorname{diag}\left(0, \mu_{2}, \ldots, \mu_{N}\right)$. Because $W$ is stochastic, we know that $1=\mu_{1} \geq \mu_{2} \geq \cdots \geq$ $\mu_{N} \geq-1$, and so $\|W-J\|=\max \left\{\left|\mu_{2}\right|,\left|\mu_{N}\right|\right\} \leq 1$.

We now show that $\mathcal{P}_{k}$ is indeed a partition. Note first that (at least) one of the events $\mathcal{A}_{0}, \ldots, \mathcal{A}_{k}$ necessarily occurs. It remains to show that the events $\mathcal{A}_{s}$ are disjoint. We carry out this by fixing arbitrary $s=1, \ldots, k$, and showing that, if the event $\mathcal{A}_{s}$ occurs, then $\mathcal{A}_{t}, t \neq s$, does not occur. Suppose that $\mathcal{A}_{s}$ occurs, i.e., the realizations $W(1), \ldots, W(k)$ are such that $\|\Phi(k, s)-J\| \leq \epsilon$ and $\|\Phi(k, s+1)-J\|>\epsilon$. Fix any $t>s$. Then, event $\mathcal{A}_{t}$ does not occur, because, by Lemma $7, \| \Phi(k, t)-$ $J\|\geq\| \Phi(k, s+1)-J \|>\epsilon$. Now, fix any $t<s$. Then, event $\mathcal{A}_{t}$ does not occur, because, by Lemma $7,\|\Phi(k, t+1)-J\| \leq$ $\|\Phi(k, s)-J\| \leq \epsilon$. Thus, for any $s=1, \ldots, k$, if the event $\mathcal{A}_{s}$ occurs, then $\mathcal{A}_{t}$, for $t \neq s$, does not occur, and hence the events $\mathcal{A}_{s}$ are disjoint.

Using the total probability law over $\mathcal{P}_{k}$, the expectation (29) is computed by:

$$
\begin{aligned}
\mathbb{E}\left[e^{\sum_{t=1}^{k} \sum_{j=1}^{N} \Lambda_{0}\left(N \lambda \Phi_{i, j}(k, t)\right)}\right] \\
=\sum_{s=0}^{k} \mathbb{E}\left[e^{\sum_{t=1}^{k} \sum_{j=1}^{N} \Lambda_{0}\left(N \lambda \Phi_{i, j}(k, t)\right)} \mathcal{I}_{\mathcal{A}_{s}}\right],
\end{aligned}
$$

where, we recall, $\mathcal{I}_{\mathcal{A}_{s}}$ is the indicator function of the event $\mathcal{A}_{s}$. The following lemma explains how to use the partition $\mathcal{P}_{k}$ to upper bound the expectation in (30).

Lemma 8: Let Assumptions 1-3 hold. Then:

(a) For any realization of the random matrices $W(t), t=$ $1,2, \ldots, k$ :

$$
\sum_{j=1}^{N} \Lambda_{0}\left(N \lambda \Phi_{i, j}(k, t)\right) \leq \Lambda_{0}(N \lambda), \forall t=1, \ldots, k .
$$

(b) Further, consider a fixed $s$ in $\{0,1, \ldots, k\}$. If the event $\mathcal{A}_{s}$ occurred, then, for $i=1, \ldots, N: \Lambda_{0}\left(N \lambda \Phi_{i, j}(k, t)\right) \leq$ $\max \left(\Lambda_{0}(\lambda-\epsilon N \sqrt{N} \lambda), \Lambda_{0}(\lambda+\epsilon N \sqrt{N} \lambda)\right), \forall t=$ $1, \ldots, s, \forall j=1, \ldots, N$.

Proof: To prove part (a) of the Lemma, by convexity of $\Lambda_{0}$, the maximum of $\sum_{j=1}^{N} \Lambda_{0}\left(N \lambda a_{j}\right)$ over the simplex $\left\{a \in \mathbb{R}^{N}: \sum_{j=1}^{N} a_{j}=1, a_{j} \geq 0, j=1, \ldots, N\right\}$ is achieved at a corner point of the simplex. The maximum equals: $\Lambda_{0}(N \lambda)+(N-1) \Lambda_{0}(0)=\Lambda_{0}(N \lambda)$, where we use the property from Lemma 1, part (b), that $\Lambda_{0}(0)=0$. Finally, since for any realization of the matrices $W(1), \ldots, W(k)$, the set of entries $\left\{\Phi_{i, j}(k, t): j=1, \ldots, N\right\}$ is a point in the simplex, the claim of part (1) of the Lemma follows.

To prove part (b) of the Lemma, suppose that event $\mathcal{A}_{s}$ occurred. Then, by the definition of $\mathcal{A}_{s}$,

$$
\|\Phi(k, s)-J\|=\|W(k) \cdots W(s)-J\| \leq \epsilon .
$$

Now, by Lemma 7:

$$
\|\Phi(k, t)-J\|=\|W(k) \cdots W(t)-J\| \leq \epsilon,
$$

for every $t \leq s$. Then, by the equivalence of the 1 -norm and the spectral norm, it follows that:

$\left|\Phi_{i, j}(k, t)-\frac{1}{N}\right| \leq \sqrt{N} \epsilon$,

$$
\text { for } t=1, \ldots, s \text {, for all } i, j=1, \ldots, N \text {. }
$$

Finally, since $\Lambda_{0}$ is convex (Lemma 1, part (a)), its maximum in $[\lambda-\epsilon N \sqrt{N} \lambda, \lambda+\epsilon N \sqrt{N} \lambda]$ is attained at a boundary point and the claim follows.

We now fix $\delta \in(0,|\log r|)$. Using the results from Lemma 4 and Lemma 8, we next bound the expectation in (30) as follows:

$$
\begin{aligned}
& \sum_{s=0}^{k} \mathbb{E}\left[e^{\sum_{t=1}^{k} \sum_{j=1}^{N} \Lambda_{0}\left(N \lambda \Phi_{i, j}(k, t)\right)} \mathcal{I}_{\mathcal{A}_{s}}\right] \\
& \leq \sum_{s=0}^{k}\left(e^{s N \max \left(\Lambda_{0}(\lambda-\epsilon N \sqrt{N} \lambda), \Lambda_{0}(\lambda+\epsilon N \sqrt{N} \lambda)\right)+(k-s) \Lambda_{0}(N \lambda)}\right) \\
& \quad \times\left(C(\delta) e^{-(k-(s+1))(\log r \mid-\delta)}\right) .
\end{aligned}
$$

To simplify the notation, we introduce the function:

$$
\begin{aligned}
g_{0}: \mathbb{R}^{2} \longrightarrow \mathbb{R} \\
g_{0}(\epsilon, \lambda):=\max \left(\Lambda_{0}(\lambda-\epsilon N \sqrt{N} \lambda), \Lambda_{0}(\lambda+\epsilon N \sqrt{N} \lambda)\right) .
\end{aligned}
$$


We need the following property of $g_{0}(\cdot, \cdot)$.

Lemma 9: Consider $g_{0}(\cdot, \cdot)$ in (32). Then, for every $\lambda \in \mathbb{R}$, the following holds:

$$
\inf _{\epsilon>0} g_{0}(\epsilon, \lambda)=\Lambda_{0}(\lambda)
$$

Proof: Since $\Lambda_{0}(\cdot)$ is convex, for $\epsilon^{\prime}<\epsilon$ and for a fixed $\lambda$, we have that

$$
\begin{aligned}
g_{0}(\epsilon, \lambda) & =\max _{\delta \in[-\epsilon, \epsilon]} \Lambda_{0}(\lambda+\delta N \sqrt{N} \lambda) \\
& \geq \max _{\delta \in\left[-\epsilon^{\prime}, \epsilon^{\prime}\right]} \Lambda_{0}(\lambda+\delta N \sqrt{N} \lambda) \\
& =g_{0}\left(\epsilon^{\prime}, \lambda\right) .
\end{aligned}
$$

Thus, for a fixed $\lambda, f(\cdot, \lambda)$ is non-increasing, and the claim of the Lemma follows.

We now bound the right hand side in (31). Rewrite $e^{-(k-(s+1))(|\log r|-\delta)}$ as $\frac{1}{r^{\delta}} e^{-(k-s)\left({ }^{\prime} \log r \mid-\delta\right)}$ : See equation (33) at the bottom of the page. The second inequality follows by introducing $\theta:=\frac{s}{k}$ and by enlarging the set for $\theta$ from $\left\{0, \frac{1}{k}, \ldots, 1\right\}$ to the continuous interval $[0,1]$. Taking the $\log$ and dividing by $k$, from (27) and (33) we get:

$$
\begin{aligned}
& \frac{1}{k} \log \alpha_{i}(k, \gamma) \leq \frac{\log (k+1)}{k}+\frac{\log \frac{C(\delta)}{r e^{\delta}}}{k} \\
& \quad+\max \left\{N g_{0}(\epsilon, \lambda), \Lambda_{0}(N \lambda)-(|\log r|-\delta)\right\}-N \gamma \lambda
\end{aligned}
$$

Taking the lim sup when $k \rightarrow \infty$, the first two terms in the right hand side of (34) vanish; further, changing the sign, we get a bound on the exponent of $\alpha_{i}(k)$ that holds for every $\epsilon \in(0,1)$ :

$$
\begin{aligned}
\liminf & -\frac{1}{k} \log \alpha_{i}(k, \gamma) \geq \\
& -\max \left\{N g_{0}(\epsilon, \lambda), \Lambda_{0}(N \lambda)-(|\log r|-\delta)\right\}+N \gamma \lambda .
\end{aligned}
$$

By Lemma 9, as $\epsilon \rightarrow 0, N g_{0}(\epsilon, \lambda)$ decreases to $N \Lambda_{0}(\lambda)$; further, letting $\delta \rightarrow 0$, we get

$$
\begin{aligned}
\liminf & -\frac{1}{k} \log \alpha_{i}(k, \gamma) \\
& \geq-\max \left\{N \Lambda_{0}(\lambda), \Lambda_{0}(N \lambda)-|\log r|\right\}+N \gamma \lambda .
\end{aligned}
$$

The previous bound on the exponent of the probability of false alarm holds for any $\lambda \geq 0$. To get the best bound, we maximize the expression on the right hand side of (35) over $\lambda \in[0, \infty)$. (We refer to Fig. 1, top and bottom, to help us illustrate the bounds $B_{0}(\gamma)$ and $B_{1}(\gamma)$ for the discrete valued observations
$Y_{i}(t)$ over a 5-point alphabet.) More precisely, we calculate $B_{0}(\gamma)$ from Theorem 5:

$$
B_{0}(\gamma)=\max _{\lambda \geq 0}\left\{N \gamma \lambda-\Phi_{0}(\lambda)\right\}
$$

where

$$
\Phi_{0}(\lambda):=\max \left\{N \Lambda_{0}(\lambda), \Lambda_{0}(N \lambda)-|\log r|\right\} .
$$

To calculate $B_{0}(\gamma)$ in (36), we need to find an optimizer $\lambda^{\star}=$ $\lambda^{\star}(\gamma)$ (if it exists) of the objective in (36); from the first order optimality conditions, $\lambda^{\star}$ is a point that satisfies:

$$
N \gamma \in \partial \Phi_{0}\left(\lambda^{\star}\right), \quad \lambda^{\star} \geq 0
$$

where $\partial \Phi_{0}(\lambda)$ denotes the subdifferential set of $\Phi_{0}$ at $\lambda$. We next characterize $\partial \Phi_{0}(\lambda)$, for $\lambda \geq 0$. Recall the zero $\lambda_{0}^{\mathrm{s}}$ of $\Delta_{0}(\cdot)$ from Theorem 5. The function $\Phi_{0}(\lambda)$ in (37) equals: 1$)$ $N \Lambda_{0}(\lambda)$ on $\lambda \in\left[0, \lambda_{0}^{\mathrm{s}}\right)$; 2) $N \Lambda_{0}\left(\lambda_{0}^{\mathrm{s}}\right)=\Lambda_{0}\left(N \lambda_{0}^{\mathrm{s}}\right)-|\log r|$ at $\lambda=\lambda_{0}^{\mathrm{s}}$; and 3) $\Lambda_{0}(N \lambda)-|\log r|$ on $\lambda>\lambda_{0}^{\mathrm{s}}$. Thus, by the rule for the subdifferential of a pointwise maximum of two convex functions, the subdifferential $\partial \Phi_{0}(\lambda)$ is:

$$
\partial \Phi_{0}(\lambda)= \begin{cases}\left\{N \Lambda_{0}^{\prime}(\lambda)\right\}, & \text { for } \lambda \in\left[0, \lambda_{0}^{\mathrm{s}}\right) \\ {\left[N \Lambda_{0}^{\prime}(\lambda), N \Lambda_{0}^{\prime}(N \lambda)\right],} & \text { for } \lambda=\lambda_{0}^{\mathrm{s}} \\ \left\{N \Lambda_{0}^{\prime}(N \lambda)\right\}, & \text { for } \lambda>\lambda_{0}^{\mathrm{s}} .\end{cases}
$$

Geometrically, $\partial \Phi_{0}(\lambda)$ is the set of slopes of the tangent lines to the graph of $\Phi_{0}(\cdot)$ at the point $\lambda$ (see Fig. 1, bottom). We next find $B_{0}(\gamma)$ for any $\gamma \in\left(\gamma_{0}, \gamma_{1}\right)$, by finding $\lambda^{\star}=\lambda^{\star}(\gamma)$ for any $\gamma \in\left(\gamma_{0}, \gamma_{1}\right)$. Geometrically, from Fig. 1 , bottom, given a slope $\gamma \in\left(\gamma_{0}, \gamma_{1}\right)$, finding a $\lambda^{\star}$ corresponds to finding a point at which a tangent line to the graph of $\Phi_{0}(\cdot)$ has a slope $\gamma$. Recall $\gamma_{0}^{-}$and $\gamma_{0}^{+}$from Theorem 5. We consider separately three regions: 1) $\gamma \in\left[\gamma_{0}, \gamma_{0}^{-}\right]$; 2) $\gamma \in\left(\gamma_{0}^{-}, \gamma_{0}^{+}\right)$; and 3) $\gamma \in\left[\gamma_{0}^{+}, \gamma_{1}\right]$. For the first region, (38) reduces to finding $\lambda^{\star} \geq 0$ such that $\gamma=\Lambda_{0}^{\prime}\left(\lambda^{\star}\right)$. Recall that $\Lambda_{0}^{\prime}(0)=\gamma_{0}$, i.e., for $\gamma=\gamma_{0}$, (38) holds (only) for $\lambda^{\star}=0$. Also, for $\gamma=\gamma_{0}^{-}$, we have, by definition of $\gamma_{0}^{-}$, that $\Lambda_{0}^{\prime}\left(\lambda_{0}^{\mathrm{s}}\right)=\gamma_{0}^{-}$, i.e., (38) holds (only) for $\lambda^{\star}=\lambda_{0}^{\mathrm{s}}$. Because $\Lambda_{0}^{\prime}(\lambda)$ is continuous and strictly increasing on $\lambda \in\left[0, \lambda_{0}^{\mathrm{s}}\right]$, it follows that, for any $\gamma \in\left[\gamma_{0}, \gamma_{0}^{-}\right]$, there exists a solution $\lambda^{\star}$ to (38), it is unique, and lies in $\left[0, \lambda_{0}^{\mathrm{s}}\right]$. Now, we calculate $B_{0}(\gamma)$ :

$$
\begin{aligned}
B_{0}(\gamma) & =N \lambda^{\star} \gamma-\Phi_{0}\left(\lambda^{\star}\right)=N \lambda^{\star} \gamma-N \Lambda_{0}\left(\lambda^{\star}\right) \\
& =N\left(\lambda^{\star} \gamma-\Lambda_{0}\left(\lambda^{\star}\right)\right)=N \sup _{\lambda \geq 0}\left(\lambda \gamma-\Lambda_{0}(\lambda)\right) \\
& =N I_{0}(\gamma)
\end{aligned}
$$

$$
\begin{aligned}
& \sum_{s=0}^{k} \frac{C(\delta)}{r e^{\delta}} e^{s N g_{0}(\epsilon, \lambda)+(k-s) \Lambda_{0}(N \lambda)-(k-s)(|\log r|-\delta)} \\
& \quad \leq(k+1) \max _{s \in\{0, \ldots, k\}} \frac{C(\delta)}{r e^{\delta}} e^{\left[s N g_{0}(\epsilon, \lambda)+(k-s)\left(\Lambda_{0}(N \lambda)-(|\log r|-\delta)\right)\right]} \\
& \quad=(k+1) \frac{C(\delta)}{r e^{\delta}} e^{\max _{s \in\{0, \ldots, k\}}\left[s N g_{0}(\epsilon, \lambda)+(k-s)\left(\Lambda_{0}(N \lambda)-\left(\mid \log r^{\prime}-\delta\right)\right)\right]} \\
& \quad \leq(k+1) \frac{C(\delta)}{r e^{\delta}} e^{k \max _{\theta \in[0,1]}\left[\theta N g_{0}(\epsilon, \lambda)+(1-\theta)\left(\Lambda_{0}(N \lambda)-(|\log r|-\delta)\right)\right]} \\
& \quad=(k+1) \frac{C(\delta)}{r e^{\delta}} e^{k \max \left\{N g_{0}(\epsilon, \lambda), \Lambda_{0}(N \lambda)-(|\log r|-\delta)\right\}}
\end{aligned}
$$


where we used the fact that $\Phi_{0}\left(\lambda^{\star}\right)=N \Lambda_{0}\left(\lambda^{\star}\right)$ (because $\lambda^{\star} \leq$ $\left.\lambda_{0}^{\mathrm{s}}\right)$, and the definition of the function $I_{0}(\cdot)$ in (12). We now consider the second region. Fix $\gamma \in\left(\gamma_{0}^{-}, \gamma_{0}^{+}\right)$. It is trivial to verify, from (39), that $\lambda^{\star}=\lambda_{0}^{\mathrm{s}}$ is the solution to (38). Thus, we calculate $B_{0}(\gamma)$ as follows:

$$
\begin{aligned}
B_{0}(\gamma) & =N \lambda_{0}^{\mathrm{s}} \gamma-\Phi_{0}\left(\lambda_{0}^{\mathrm{s}}\right)=N \lambda_{0}^{\mathrm{s}} \gamma-N \Lambda_{0}\left(\lambda_{0}^{\mathrm{s}}\right) \\
& =N \lambda_{0}^{\mathrm{s}}\left(\gamma-\gamma_{0}^{-}\right)+N \lambda_{0}^{\mathrm{s}} \gamma_{0}^{-}-N \Lambda_{0}\left(\lambda_{0}^{\mathrm{s}}\right) \\
& =N \lambda_{0}^{\mathrm{s}}\left(\gamma-\gamma_{0}^{-}\right)+N I_{0}\left(\gamma_{0}^{-}\right),
\end{aligned}
$$

where we used the fact that $\lambda_{0}^{\mathrm{s}} \gamma_{0}^{-}-\Lambda_{0}\left(\lambda_{0}^{\mathrm{s}}\right)=\sup _{\lambda \geq 0} \lambda \gamma_{0}^{-}-$ $\Lambda_{0}(\lambda)=I_{0}\left(\gamma_{0}^{-}\right)$. The proof for the third region is analogous to the proof for the first region.

For a proof of the claim on the probability of miss $\beta_{i}(k, \gamma)=$ $\mathbb{P}\left(x_{i}(k)<\gamma \mid H_{1}\right)$, we proceed analogously to (26), where instead of $\zeta \geq 0$, we now use $\zeta \leq 0$ (and, hence, the proof proceeds with $\lambda \leq 0$ ).

Proof of Corollary 6: We first prove part (a). Consider the error probability $P_{\mathrm{e}, \mathrm{i}}(k, \gamma)$ in (19). By Lemma 1.2.15 in [31], we have that:

$$
\begin{aligned}
& \liminf _{k \rightarrow \infty}-\frac{1}{k} \log P_{\mathrm{e}, \mathrm{i}}(k, \gamma) \\
& =\min \left\{\liminf _{k \rightarrow \infty}-\frac{1}{k} \log \left(\alpha_{i}(k, \gamma) \pi_{0}\right),\right. \\
& \left.\liminf _{k \rightarrow \infty}-\frac{1}{k} \log \left(\beta_{i}(k, \gamma) \pi_{1}\right)\right\} \\
& =\min \left\{\liminf _{k \rightarrow \infty}-\frac{1}{k} \log \alpha_{i}(k, \gamma),\right. \\
& \left.\geq \operatorname{limin}\left\{B_{0}(\gamma), B_{1}(\gamma)\right\}, \frac{1}{k} \log \beta_{i}(k, \gamma)\right\}
\end{aligned}
$$

where the last inequality is by Theorem 5; thus, the left inequality in (24). We now show the right inequality in (24), i.e., $\min \left\{B_{0}(\gamma), B_{1}(\gamma)\right\}>0$ for all $\gamma \in\left(\gamma_{0}, \gamma_{1}\right)$. First, from the expression for $B_{0}(\gamma)$ in Theorem 5 , for $|\log r|>0$, we have: $B_{0}\left(\gamma_{0}\right)=N I_{0}\left(\gamma_{0}\right)=0$, and $B_{0}^{\prime}(\gamma)=N I_{0}^{\prime}(\gamma)>0$ for any $\gamma \in\left(\gamma_{0}, \gamma_{0}^{-}\right)$. As the function $B_{0}(\cdot)$ is convex, we conclude that $B_{0}(\gamma)>0$, for all $\gamma>\gamma_{0}$. (The same conclusion holds under $|\log r|=0$, by replacing $N I_{0}(\gamma)$ with $I_{0}(\gamma)+|\log r|=I_{0}(\gamma)$.) Analogously, it can be shown that $B_{1}(\gamma)>0$ for all $\gamma<\gamma_{1}$; thus, $\min \left\{B_{0}(\gamma), B_{1}(\gamma)\right\}>0$, for all $\gamma \in\left(\gamma_{0}, \gamma_{1}\right)$.

We now calculate $\max _{\gamma \in\left(\gamma_{0}, \gamma_{1}\right)} \min \left\{B_{0}(\gamma), B_{1}(\gamma)\right\}$. Consider the function $\Delta_{B}(\gamma):=B_{1}(\gamma)-B_{0}(\gamma)$. Using the definition of $B_{0}(\gamma)$ in Theorem 5, and taking the subdifferential of $B_{0}(\gamma)$ at any point $\gamma \in\left(\gamma_{0}, \gamma_{1}\right)$, it is easy to show that $B_{0}^{\prime}(\gamma)>0$, for any subgradient $B_{0}^{\prime}(\gamma) \in \partial B_{0}(\gamma)$, which implies that $B_{0}(\cdot)$ is strictly increasing on $\gamma \in\left(\gamma_{0}, \gamma_{1}\right)$. Similarly, it can be shown that $B_{1}(\cdot)$ is strictly decreasing on $\gamma \in\left(\gamma_{0}, \gamma_{1}\right)$. Further, using the properties that $I_{0}\left(\gamma_{0}\right)=0$ and $I_{1}\left(\gamma_{1}\right)=0$, we have $\Delta_{B}\left(\gamma_{0}\right)=B_{1}\left(\gamma_{0}\right)>0$, and $\Delta_{B}\left(\gamma_{1}\right)=-B_{0}\left(\gamma_{1}\right)<0$. By the previous two observations, we have that $\Delta_{B}(\gamma)$ is strictly decreasing on $\gamma \in\left(\gamma_{0}, \gamma_{1}\right)$, with $\Delta_{B}\left(\gamma_{0}\right)>0$ and $\Delta_{B}\left(\gamma_{1}\right)<0$. Thus, $\Delta_{B}(\cdot)$ has a unique zero $\gamma^{\star}$ on $\left(\gamma_{0}, \gamma_{1}\right)$. Now, the claim: $\max _{\gamma \in\left(\gamma_{0}, \gamma_{1}\right)} \min \left\{B_{0}(\gamma), B_{1}(\gamma)\right\}=B_{0}\left(\gamma^{\star}\right)=B_{1}\left(\gamma^{\star}\right)$ holds trivially because $B_{0}(\cdot)$ is strictly increasing on $\gamma \in\left(\gamma_{0}, \gamma_{1}\right)$,
$B_{1}(\cdot)$ is strictly decreasing on $\gamma \in\left(\gamma_{0}, \gamma_{1}\right)$, and $B_{0}(\cdot)$ and $B_{1}(\cdot)$ intersect at $\gamma^{\star} \in\left(\gamma_{0}, \gamma_{1}\right)$. This completes the proof of part (a).

We now prove part (b). Suppose that $|\log r| \geq \operatorname{thr}\left(\Lambda_{0}, N\right)$. We show that, for $\gamma=0$ :

$$
B_{0}(0)=N I_{0}(0), B_{1}(0)=N I_{1}(0)=N I_{0}(0) .
$$

(The last equality in (44) holds because $I_{1}(0)=\left(I_{0}(\gamma)-\right.$ $\gamma)\left.\right|_{\gamma=0}=I_{0}(0)$.)

We prove only the equality for $B_{0}$ in (44) as the equality for $B_{1}$ follows similarly. Because $|\log r| \geq \operatorname{thr}\left(\Lambda_{0}, N\right)$, we have, by the definition of $\Phi_{0}(\cdot)$ in (37), that $\Phi_{0}\left(\lambda^{\bullet}\right)=N \Lambda_{0}\left(\lambda^{\bullet}\right)$. Recall that $B_{0}(0)=-\Phi_{0}\left(\lambda^{\star}\right)$, where $\lambda^{\star}$ is a point for which (38) holds for $\gamma=0$. However, because $\partial \Phi_{0}\left(\lambda^{\bullet}\right)=\left\{N \Lambda_{0}^{\prime}\left(\lambda^{\bullet}\right)\right\}$, and $\Lambda_{0}^{\prime}\left(\lambda^{\bullet}\right)=0$, it follows that $\lambda^{\star}=\lambda^{\bullet}$ and $B_{0}(0)=-\Phi_{0}\left(\lambda^{\bullet}\right)=-N \Lambda_{0}\left(\lambda^{\bullet}\right)=N I_{0}(0)$, which proves (44).

Now, (44) means that $B_{0}(0)=B_{1}(0)$. Further, $0 \in\left(\gamma_{0}, \gamma_{1}\right)$, and, from part (a), $\gamma^{\star}$ is unique, and so $\gamma^{\star}$ has to be 0 . This shows that $\sup _{\gamma \in\left(\gamma_{0}, \gamma_{1}\right)} \min \left\{B_{0}(\gamma), B_{1}(\gamma)\right\}=N I_{0}(0)=N C_{\text {ind }}$, and so, by part (a):

$$
\liminf _{k \rightarrow \infty}-\frac{1}{k} \log P_{\mathrm{e}, \mathrm{i}}(k, 0) \geq N C_{\text {ind }} .
$$

On the other hand,

$$
\limsup _{k \rightarrow \infty}-\frac{1}{k} \log P_{\mathrm{e}, \mathrm{i}}(k, 0) \leq N C_{\text {ind }},
$$

because, by the Chernoff lemma [31], for any test (with the corresponding error probability $P_{\mathrm{e}}^{\prime}(k, \gamma)$,) we have that $\lim \sup _{k \rightarrow \infty}-\frac{1}{k} \log P_{\mathrm{e}}^{\prime}(k, \gamma) \leq N C_{\text {ind }}$. Combining (45) and (46) yields'

$$
\begin{aligned}
N C_{\text {ind }} & \leq \liminf _{k \rightarrow \infty}-\frac{1}{k} \log P_{\mathrm{e}, \mathrm{i}}(k, 0) \\
& \leq \limsup _{k \rightarrow \infty}-\frac{1}{k} \log P_{\mathrm{e}, \mathrm{i}}(k, 0) \leq N C_{\text {ind }} .
\end{aligned}
$$

Thus, the result in part (b) of the Lemma holds.

\section{EXAMPLES}

This section illustrates our main results for several examples of the distributions of the sensor observations. Section IV-A compares the Gaussian and Laplace distributions, both with a finite number of sensors $N$ and when $N \rightarrow \infty$. Section IV-B considers discrete distributions with finite support, and, in more detail, binary distributions. Finally, Section IV-C numerically demonstrates that our theoretical lower bound on the error exponent (24) is tight. Section IV-C also shows through a symmetric, tractable example how distributed detection performance depends on the network topology (sensors' degree and link occurrence/failure probability.)

\section{A. Gaussian Distribution Versus Laplace Distribution}

Gaussian distribution. We now study the detection of a signal in additive Gaussian noise; $Y_{i}(t)$ has the following density:

$$
f_{\mathrm{G}}(y)= \begin{cases}\frac{1}{\sqrt{2 \pi} \sigma_{\mathrm{G}}} e^{-\frac{\left(y-m_{\mathrm{G}}\right)^{2}}{2 \sigma_{\mathrm{G}}^{2}}}, & H_{1} \\ \frac{1}{\sqrt{2 \pi} \sigma_{\mathrm{G}}} e^{-\frac{y^{2}}{2 \sigma_{\mathrm{G}}^{2}}}, & H_{0} .\end{cases}
$$


The LMGF is given by: $\Lambda_{0, \mathrm{G}}(\lambda)=-\frac{\lambda(1-\lambda)}{2} \frac{m_{\mathrm{G}}^{2}}{\sigma_{\mathrm{G}}^{2}}$. The minimum of $\Lambda_{0, \mathrm{G}}$ is achieved at $\lambda^{\bullet}=\frac{1}{2}$, and the per sensor Chernoff information is

$$
C_{\text {ind, } \mathrm{G}}=\frac{m_{\mathrm{G}}^{2}}{8 \sigma_{\mathrm{G}}^{2}} .
$$

Applying Corollary 6 (b), we get the sufficient condition for optimality:

$$
\begin{aligned}
|\log r| & \geq \Lambda_{0, \mathrm{G}}\left(\frac{N}{2}\right)-N \Lambda_{0, \mathrm{G}}\left(\frac{1}{2}\right) \\
& =N(N-1) C_{\text {ind, } \mathrm{G} .}
\end{aligned}
$$

Since $\Lambda_{0}(\lambda)=\Lambda_{1}(\lambda)$, the two conditions from the Corollary here reduce to a single condition in (24).

Now, let the number of sensors $N \rightarrow \infty$, while keeping the total Chernoff information constant, i.e., not dependent on $N$; that is, $C_{\mathrm{G}}:=N C_{\mathrm{ind}, \mathrm{G}}=$ const, $C_{\mathrm{ind}, \mathrm{G}}(N)=\frac{C_{\mathrm{G}}}{N}$. Intuitively, as $N$ increases, we deploy more and more sensors over a region (denser deployment), but, on the other hand, the sensors' quality becomes worse and worse. The increase of $N$ is balanced in such a way that the total information offered by all sensors stays constant with $N$. Our goal is to determine how the optimality threshold on the network connectivity $\operatorname{thr}\left(N, \Lambda_{0, \mathrm{G}}\right)$ depends on $N$. We can see from (47) that the optimality threshold for the distributed detector in the Gaussian case equals:

$$
\operatorname{thr}\left(\Lambda_{0, \mathrm{G}}, N\right)=(N-1) C_{\mathrm{G}} .
$$

Laplace distribution. We next study the optimality conditions for the sensor observations with Laplace distribution. The density of $Y_{i}(t)$ is:

$$
f_{\mathrm{L}}(y)= \begin{cases}\frac{1}{2 b_{\mathrm{L}}} e^{-\frac{\left|y-m_{\mathrm{L}}\right|}{b_{\mathrm{L}}}}, & H_{1} \\ \frac{1}{2 b_{\mathrm{L}}} e^{-\frac{|y|}{b_{\mathrm{L}}}}, & H_{0} .\end{cases}
$$

The LMGF has the following form:

$$
\Lambda_{0, \mathrm{~L}}(\lambda)=\log \left(\frac{1-\lambda}{1-2 \lambda} e^{-\lambda \frac{m_{\mathrm{L}}}{b_{\mathrm{L}}}}-\frac{\lambda}{1-2 \lambda} e^{-(1-\lambda) \frac{m_{\mathrm{L}}}{b_{\mathrm{L}}}}\right) .
$$

Again, the minimum is at $\lambda^{\bullet}=\frac{1}{2}$; the per sensor Chernoff information is $C_{\text {ind, } \mathrm{L}}=\frac{m_{\mathrm{L}}}{2 b_{\mathrm{L}}}-\log \left(1+\frac{m_{\mathrm{L}}}{2 b_{\mathrm{L}}}\right)$. The optimality condition in (24) becomes:

$$
\begin{aligned}
|\log r| \geq & \Lambda_{0, \mathrm{~L}}\left(\frac{N}{2}\right)-N \Lambda_{0, \mathrm{~L}}\left(\frac{1}{2}\right) \\
= & \log \left(\frac{2-N}{2-2 N} e^{-\frac{N}{2} \frac{m_{\mathrm{L}}}{b_{\mathrm{L}}}}-\frac{N}{2-2 N} e^{-\left(1-\frac{N}{2}\right) \frac{m_{\mathrm{L}}}{b_{\mathrm{L}}}}\right) \\
& -N \log \left(1+\frac{m_{\mathrm{L}}}{2 b_{\mathrm{L}}}\right)+N \frac{m_{\mathrm{L}}}{2 b_{\mathrm{L}}}
\end{aligned}
$$

Gaussian versus Laplace distribution. It is now interesting to compare the Gaussian and the Laplace case under equal per sensor Chernoff information $C_{\text {ind, }}=C_{\text {ind, G }}$. Fig. 2 (top) plots the LMGF for the Gaussian and Laplace distributions, for $N=$ $10, C_{\text {ind }}=C_{\text {ind }, \mathrm{L}}=C_{\text {ind }, \mathrm{G}}=0.0945, b_{\mathrm{L}}=1, m_{\mathrm{L}}=1$, and $\frac{m_{G}^{2}}{\sigma_{\mathrm{G}}^{2}}=0.7563=8 C_{\text {ind }}$. By (25), the optimality threshold equals

$$
\left|N \Lambda_{0}\left(\frac{1}{2}\right)\right|+\left|\Lambda_{0}\left(\frac{N}{2}\right)\right|
$$
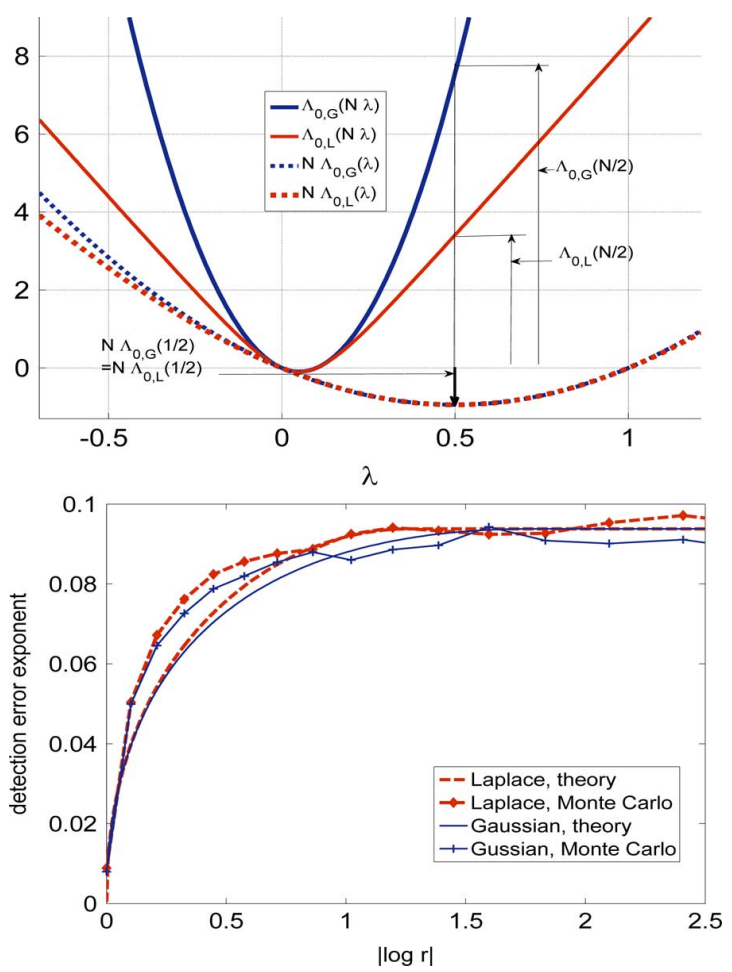

Fig. 2. Top: LMGFs for Gaussian and Laplace distributions with equal per sensor Chernoff informations, for $N=10, C_{\text {ind }}=C_{\text {ind }}, \mathrm{L}=C_{\text {ind }}, \mathrm{G}=$ $0.0945, b_{\mathrm{L}}=1, m_{\mathrm{L}}=1$, and $\frac{m_{\mathrm{G}}^{2}}{\sigma_{\mathrm{G}}^{2}}=0.7563=8 C_{\mathrm{ind}}$. Solid lines plot the functions $\Lambda_{0}(N \lambda)$ for the two distributions, while dashed lines plot the functions $N \Lambda_{0}(\lambda)$. For both solid and dashed lines, the Gaussian distribution corresponds to the more curved functions. The optimality threshold in (25) is given by $\left|N \Lambda_{0}\left(\frac{1}{2}\right)\right|+\left|\Lambda_{0}\left(\frac{N}{2}\right)\right|$, as $\lambda^{\bullet}=\frac{1}{2}$. Bottom: Lower bound on the error exponent in (24) and the Monte Carlo estimate of the error exponent versus $\mid \log r$ for the Gaussian and Laplace sensor observations: $N=20, C_{\text {ind }}=C_{\text {ind }}, \mathrm{L}=$ $C_{\text {ind }}, \mathrm{G}=0.005, b_{\mathrm{L}}=1, m_{\mathrm{L}}=0.2$, and $\frac{m_{\mathrm{G}}^{2}}{\sigma_{\mathrm{G}}^{2}}=0.04=8 C_{\text {ind }}$.

as $\lambda^{\bullet}=\frac{1}{2}$, for both the Gaussian and Laplace distributions. The threshold can be estimated from Fig. 2 (top): solid lines plot the functions $\Lambda_{0}(N \lambda)$ for the two different distributions, while dashed lines plot the functions $N \Lambda_{0}(\lambda)$. For both solid and dashed lines, the Gaussian distribution corresponds to the more curved functions. We see that the threshold is larger for the Gaussian case. This means that, for a certain range $r \in$ $\left(r_{\min }, r_{\max }\right)$, the distributed detector with Laplace sensors is asymptotically optimal, while with Gaussian sensors the distributed detector may not be optimal, even though it uses the same network infrastructure (same $r$ ) and has same per sensor Chernoff information. (See also Fig. 2 (bottom) for another illustration of this effect.)

We now compare the Gaussian and Laplace distributions when $N \rightarrow \infty$, and we keep the Gaussian total Chernoff information $C_{\mathrm{G}}$ constant with $N$. Let the Laplace distribution parameters vary with $N$ as:

$$
m_{\mathrm{L}}=m_{\mathrm{L}}(N)=\frac{2 \sqrt{2 C_{\mathrm{G}}}}{\sqrt{N}}, b_{\mathrm{L}}=b_{\mathrm{L}}(N)=1 .
$$

We can show that, as $N \rightarrow \infty$, the total Chernoff information $C_{\mathrm{L}}(N) \rightarrow C_{\mathrm{G}}$ as $N \rightarrow \infty$, and so the Gaussian and the Laplace centralized detectors become equivalent. On the other hand, the threshold for the Gaussian distributed detector is given by (48), 
while, for the Laplace detector, using (49) and a Taylor expansion, we get that the optimality threshold is approximately:

$$
\operatorname{thr}\left(\Lambda_{0, \mathrm{~L}}, N\right) \approx \sqrt{2 C_{\mathrm{G}} N} .
$$

Hence, the required $|\log r|$ to achieve the optimal error exponent grows much slower with the Laplace distribution than with the Gaussian distribution.

\section{B. Discrete Distributions}

We now consider the case when the support of the sensor observations under both hypotheses is a finite alphabet $\left\{a_{1}, a_{2}, \ldots, a_{M}\right\}$. This case is of practical interest when, for example, the sensing device has an analog-to-digital converter with a finite range; hence, the observations take only a finite number of values. Specifically, the distribution of $Y_{i}(k), \forall i$, $\forall k$, is given by:

$$
\mathbb{P}\left(Y_{i}(k)=a_{m}\right)=\left\{\begin{array}{ll}
q_{m}, & H_{1} \\
p_{m}, & H_{0}
\end{array}, m=1, \ldots, M .\right.
$$

Then, the LMGF under $H_{0}$ equals:

$$
\Lambda_{0}(\lambda)=\log \left(\sum_{m=1}^{M} q_{m}^{\lambda} p_{m}^{1-\lambda}\right) .
$$

Note that $\Lambda_{0}(\lambda)$ is finite on $\mathbb{R}$. Due to concavity of $-\Lambda_{0}(\cdot)$, the argument of the Chernoff information $\lambda^{\bullet}\left(C_{\text {ind }}=\right.$ $\left.\max _{\lambda_{\in[0,1]}}\left\{-\Lambda_{0}(\lambda)\right\}=-\Lambda_{0}\left(\lambda^{\bullet}\right)\right)$ can, in general, be efficiently computed numerically, for example, by the Netwon method (see, e.g., [34], for details on the Newton method.) It can be shown, defining $c_{m}=\log \left(\frac{q_{m}}{p_{m}}\right)$, that the Newton direction, e.g., [34], equals:

$$
\begin{aligned}
& d(\lambda)=-\left(\Lambda_{0}^{\prime \prime}(\lambda)\right)^{-1} \Lambda_{0}^{\prime}(\lambda) \\
& =-\frac{1}{\frac{\sum_{m=1}^{M} c_{m}^{2} p_{m} e^{\lambda c_{m}}}{\sum_{m=1}^{M} c_{m} p_{m} e^{\lambda c_{m}}}-\frac{\sum_{m=1}^{M} c_{m} p_{m} e^{\lambda c_{m}}}{\sum_{m=1}^{M} c_{m} e^{\lambda c_{m}}} .}
\end{aligned}
$$

Binary observations. To gain more intuition and obtain analytical results, we consider (50) with $M=2$, i.e., binary sensors, with $p_{2}=1-p_{1}=1-p, q_{2}=1-q_{1}=1-q$. Suppose further that $p<q$. We can show that the negative of the per sensor Chernoff information $\Lambda_{0, \text { bin }}$ and the quantity $\lambda \bullet$ are:

$$
\begin{aligned}
-C_{\text {ind }}= & \Lambda_{0, \operatorname{bin}}\left(\lambda^{\bullet}\right)=\lambda^{\bullet} \log \left(\frac{q}{p}\right)+\log p \\
& +\log \left(1-\frac{\log \frac{q}{p}}{\log \frac{1-q}{1-p}}\right), \\
\lambda^{\bullet}= & \frac{\log \frac{p}{1-p}+\log \left(\frac{\log \frac{p}{q}}{\log \frac{1-q}{1-p}}\right)}{\log \left(\frac{1-q}{1-p}\right)-\log \left(\frac{q}{p}\right)} .
\end{aligned}
$$

Further, note that:

$$
\begin{aligned}
\Lambda_{0, \text { bin }}\left(N \lambda^{\bullet}\right) & =\log \left(p\left(\frac{q}{p}\right)^{N \lambda^{\bullet}}+(1-p)\left(\frac{1-q}{1-p}\right)^{N \lambda^{\bullet}}\right) \\
& \leq \log \left(\frac{q}{p}\right)^{N \lambda^{\bullet}}=N \lambda^{\bullet} \log \left(\frac{q}{p}\right) .
\end{aligned}
$$

Also, we can show similarly that:

$$
\Lambda_{0, \text { bin }}\left(1-N\left(1-\lambda^{\bullet}\right)\right) \leq N\left(1-\lambda^{\bullet}\right) \log \left(\frac{1-p}{1-q}\right) .
$$

Combining (51) and (52), and applying Corollary 6 (24), we get that a sufficient condition for asymptotic optimality is:

$$
\begin{array}{r}
|\log r| \geq \max \left\{N \log \frac{1}{p}-N \log \left(1+\frac{\left|\log \frac{q}{p}\right|}{\left|\log \frac{1-q}{1-p}\right|}\right)\right. \\
\left.\quad N \log \frac{1}{1-q}-N \log \left(1+\frac{\log \frac{1-q}{1-p} \mid}{\left|\log \frac{q}{p}\right|}\right)\right\} .
\end{array}
$$

From the equation above, we can further obtain a very simplified sufficient condition for optimality:

$$
|\log r| \geq N \max \{|\log p|,|\log (1-q)|\} .
$$

The expression in (53) is intuitive. Consider, for example, the case $p=\frac{1}{2}$, so that the right hand side in (53) simplifies to: $N|\log (1-q)|$. Let $q$ vary from $\frac{1}{2}$ to 1 . Then, as $q$ increases, the per sensor Chernoff information increases, and the optimal centralized detector has better and better performance (error exponent.) That is, the centralized detector has a very low error probability after a very short observation interval $k$. Hence, for larger $q$, the distributed detector needs more connectivity to be able to "catch up" with the performance of the centralized detector. We compare numerically Gaussian and binary distributed detectors with equal per sensor Chernoff information, for $N=32$ sensors, $C_{\text {ind }}=5.11 \cdot 10^{-4}, \frac{m_{\mathrm{G}}^{2}}{\sigma_{\mathrm{G}}^{2}}=8 C_{\text {ind }}, p=0.1$, and $q=0.12$. Binary detector requires more connectivity to achieve asymptotic optimality $(r \approx 0.25)$, while Gaussian detector requires $r \approx 0.5$.

\section{Tightness of the Error Exponent Lower Bound in (24) and the Impact of the Network Topology}

Assessment of the tightness of the error exponent lower bound in (24). We note that the result in (24) is a theoretical lower bound on the error exponent. In particular, the condition $|\log r| \geq \operatorname{thr}\left(\Lambda_{0}, N\right)$ is proved to be a sufficient, but not necessary, condition for asymptotically optimal detection; in other words, (24) does not exclude the possibility of achieving asymptotic optimality for a certain value of $|\log r|$ smaller than $\operatorname{thr}\left(\Lambda_{0}, N\right)$. In order to assess the tightness of (24) (for both the Gaussian and Laplace distributions,) we perform Monte Carlo simulations to estimate the actual error exponent and compare it with (24). We consider $N=20$ sensors and fix the sensor observation distributions with the following parameters: $C_{\text {ind }}=$ $C_{\text {ind, } \mathrm{L}}=C_{\text {ind, } \mathrm{G}}=0.005, b_{\mathrm{L}}=1, m_{\mathrm{L}}=0.2$, and $\frac{m_{\mathrm{C}}^{2}}{\sigma_{\mathrm{C}}^{2}}=$ $0.04=8 C_{\text {ind }}$. We vary $r$ as follows. We construct a (fixed) geometric graph with $N$ sensors by placing the sensors uniformly at random on a unit square and connecting the sensors whose distance is less than a radius. Each link is a Bernoulli random variable, equal to 1 with probability $p$ (link online), and equal to 0 with probability $1-p$ (link offline). The link occurrences are independent in time and space. We change $r$ by varying $p$ from 0 to 0.95 in the increments of 0.05 . We adopt the Metropolis weights: whenever a link $\{i, j\}$ is online, we set $W_{i j}(k)=\frac{1}{\left(1+\max \left(d_{i}(k), d_{j}(k)\right)\right)}$, where $d_{i}(k)$ is the number of 
neighbors of sensor $i$ at time $k$; when a link $\{i, j\}$ is offline, $W_{i j}(k)=0$; and $W_{i i}(k)=1-\sum_{j \in O_{i}(k)} W_{i j}(k)$, where we recall that $O_{i}(k)$ is the neighborhood of sensor $i$. We obtain an estimate of the error probability $\widehat{P}_{\mathrm{e}, \mathrm{i}}(k)$ at sensor $i$ and time $k$ using 30,000 Monte Carlo runs of (13) per each hypothesis. We then estimate the sensor-wide average error exponent as:

$$
\frac{1}{N} \sum_{i=1}^{N} \frac{\log \widehat{P}_{\mathrm{e}, \mathrm{i}}\left(K_{1}\right)-\log \widehat{P}_{\mathrm{e}, \mathrm{i}}\left(K_{2}\right)}{K_{2}-K_{1}},
$$

with $K_{1}=40, K_{2}=60$. That is, we estimate the error exponent as the average slope (across sensors) of the error probability curve in a semi-log scale. Fig. 2 (bottom) plots both the theoretical lower bound on the error exponent in (24) and the Monte Carlo estimate of the error exponent versus $|\log r|$ for Gaussian and Laplace distributions. We can see that the bound (24) is tight for both distributions. Hence, the actual distributed detection performance is very close to the performance predicted by (24). Of course, above the optimality threshold, (24) and the actual error exponent coincide and are equal to the total Chernoff information. Also, we can see that the theoretical threshold by optimality $\operatorname{thr}\left(\Lambda_{0}, N\right)$ and the threshold value computed from simulation are very close. Finally, the distributed detector with Laplace observations achieves asymptotic optimality for a smaller value of $|\log r|(|\log r| \approx 1.2)$ than the distributed detector with Gaussian observations $(|\log r| \approx 1.6)$, even though the corresponding centralized detectors are asymptotically equivalent.

Impact of the network topology. We have seen in the previous two subsections how detection performance depends on $r$. In order to understand how $r$ depends on the network topology, we consider a symmetric network structure, namely a regular network. For this case, we can express $r$ as an explicit (closed form) function of the sensors' degrees and the link occurrence probabilities. Recall that the smaller $r$ is, the better the network connectivity.

Consider a connected regular network with $N$ sensors and degree $d \geq 2$. Suppose that each link is a Bernoulli random variable, equal to 1 with probability $p$ (link online) and 0 with probability $1-p$ (link offline,) with spatio-temporally independent link occurrences. Then, it can be shown [32] that $r$ equals:

$$
r=(1-p)^{d} .
$$

This expression is very intuitive. When $p$ increases, i.e., when the links are online more often, the network (on average) becomes more connected, and hence we expect that the network connectivity $|\log r|$ increases (improves). This is confirmed by (54): when $p$ increases, $r$ becomes smaller and closer to zero, and hence $|\log r|$ increases. Further, when $d$ increases, the network becomes more connected, and hence the network speed again improves. Note also that $|\log r|=d|\log (1-p)|$ is a linear function of $d$.

We now recall Corollary 6 to relate distributed detection performance with $p$ and $d$. For example, for a fixed $p$, the distributed detection optimality condition becomes $d>\frac{\operatorname{thr}\left(\Lambda_{0}, N\right)}{|\log (1-p)|}$, i.e., distributed detection is asymptotically optimal when the sensors' degree is above a threshold. Further, because $d \leq N$, it follows that, for a large value of $\operatorname{thr}\left(\Lambda_{0}, N\right)$ and a small $p$, even the networks with a very large degree (say, $d=N-1$ ) do not achieve asymptotic optimality. Intuitively, a large $\operatorname{thr}\left(\Lambda_{0}, N\right)$ means that the corresponding centralized detector decreases the error probability so fast in $k$ that, because of the intermittent link failures, the distributed detector cannot "catch up" with the centralized detector. Finally, when $p=1$, the optimality condition becomes $d>0$, i.e., distributed detection is asymptotically optimal for any $d \geq 2$. This is because, when $p=1$, the network is always connected, and the distributed detector asymptotically "catches up" with an arbitrarily fast centralized detector. In fact, it can be shown that an arbitrarily connected network with no link failures achieves asymptotic optimality for any value of $\operatorname{thr}\left(\Lambda_{0}, N\right)$. (Such a network has $r=0$ [32], and the network connectivity $|\log r|$ is $\propto$.)

\section{Non-Identically Distributed ObServations}

We extend Theorem 5 and Corollary 6 to the case of (independent) non-identically distributed observations. First, we briefly explain the measurement model and define the relevant quantities. As before, let $Y_{i}(t)$ denote the observation of sensor $i$ at time $t, i=1, \ldots, N, t=1,2, \ldots$.

Assumption A: The observations of sensor $i$ are i.i.d. in time, with the following distribution:

$$
Y_{i}(t) \sim\left\{\begin{array}{ll}
\nu_{i, 1}, & H_{1} \\
\nu_{i, 0}, & H_{0}
\end{array}, i=1, \ldots, N, t=1,2, \ldots .\right.
$$

(Here we assume that $\nu_{i, 1}$ and $\nu_{i, 0}$ are mutually absolutely continuous, distinguishable measures, for $i=1, \ldots, N)$. Further, the observations of different sensors are independent both in time and in space, i.e., for $i \neq j, Y_{i}(t)$ and $Y_{j}(k)$ are independent for all $t$ and $k$.

Under Assumption A, the form of the log-likelihood ratio test remains the same as under Assumption 1:

$$
D(k):=\frac{1}{N k} \sum_{t=1}^{k} \sum_{i=1}^{N} L_{i}(t) \underset{H_{1}}{\stackrel{H_{0}}{\gtrless}} \gamma,
$$

where the $\log$-likelihood ratio at sensor $i, i=1, \ldots, N$, is now:

$$
L_{i}(t)=\log \frac{f_{i, 1}\left(Y_{i}(t)\right)}{f_{i, 0}\left(Y_{i}(t)\right)}
$$

and $f_{i, l}, l=0,1$, is the density (or the probability mass) function associated with $\nu_{i, l}$. We now discuss the choice of detector thresholds $\gamma$. Let $\bar{\gamma}_{l}=\mathbb{E}\left[\frac{1}{N} \sum_{i=1}^{N} L_{i}(t) \mid H_{l}\right]=\frac{\left(\sum_{i=1}^{N} \gamma_{i, l}\right)}{N}$, where $\gamma_{i, l}=E\left[L_{i}(t) \mid H_{l}\right]$. We can show that, if $|\log r|>0$, then any $\gamma \in\left(\bar{\gamma}_{0}, \bar{\gamma}_{1}\right)$ yields an exponentially fast decay of the error probability, at any sensor. The condition $|\log r|>0$ means that the network is connected on average, e.g., [35]; if met, then, for all $i, \mathbb{E}\left[x_{i}(k) \mid H_{l}\right] \rightarrow \bar{\gamma}_{l}$ as $k \rightarrow \infty, l=0,1$. (Proof is omitted for brevity.) Clearly, under identical sensors, $\gamma_{i, l}=\gamma_{j, l}$ for any $i, j$, and hence the range of detector thresholds becomes the one assumed in Section II-C.

Denote by $\Lambda_{i, 0}$ the LMGF of $L_{i}(t)$ under hypothesis $H_{0}$ :

$$
\Lambda_{i, 0}: \mathbb{R} \longrightarrow(-\infty,+\infty], \Lambda_{i, 0}(\lambda)=\log \mathbb{E}\left[e^{\lambda L_{i}(1)} \mid H_{0}\right] .
$$

We assume finiteness of the LMGF's of all sensors. Assumption 2 is restated explicitly as Assumption B.

Assumption B: For $i=1, \ldots N, \Lambda_{i, 0}(\lambda)<+\infty, \forall \lambda \in \mathbb{R}$. 
The optimal centralized detector, with highest error exponent, is the log-likelihood ratio test with zero threshold $\gamma=0$ [31], its error exponent is equal to the Chernoff information of the vector of all sensors observations, and can be expressed in terms of the LMGF's as:

$$
C_{\text {tot }}=\max _{\lambda \in[0,1]}-\sum_{i=1}^{N} \Lambda_{i, 0}(\lambda)=-\sum_{i=1}^{N} \Lambda_{i, 0}\left(\lambda^{\bullet}\right) .
$$

Here, $\lambda^{\bullet}$ is the minimizer of $\sum_{i=1}^{N} \Lambda_{i, 0}$ over $[0,1]$. We are now ready to state our results on the error exponent of the consensus+innovation detector for the case of non-identically distributed observations. We continue to use $\alpha_{i}(k, \gamma), \beta_{i}(k, \gamma)$, and $P_{\mathrm{e}, \mathrm{i}}(k, \gamma)$ to denote the false alarm, miss, and Bayes error probabilities of the distributed detector at sensor $i$.

Theorem 10: Let Assumptions A, B and 3 hold, and let, in addition, $|\log r|>0$. Consider the family of distributed detectors in (13) and (14) with thresholds $\gamma \in\left(\bar{\gamma}_{0}, \bar{\gamma}_{1}\right)$. Then, at each sensor $i$ :

$$
\begin{aligned}
& \liminf _{k \rightarrow \infty}-\frac{1}{k} \log \alpha_{i}(k, \gamma) \geq B_{0}(\gamma)>0, \\
& \liminf _{k \rightarrow \infty}-\frac{1}{k} \log \beta_{i}(k, \gamma) \geq B_{1}(\gamma)>0,
\end{aligned}
$$

where

$$
\begin{aligned}
B_{0}(\gamma)= & \max _{-\lambda \in[0,1]}\{N \lambda \gamma- \\
& \left.\max \left\{\sum_{i=1}^{N} \Lambda_{i, 0}(\lambda) \max _{i=1, \ldots, N} \Lambda_{i, 0}(N \lambda)-|\log r|\right\}\right\} \\
B_{1}(\gamma)= & \max _{\lambda \in[-1,0]}\{N \lambda \gamma- \\
& \left.\max \left\{\sum_{i=1}^{N} \Lambda_{i, 1}(\lambda) \max _{i=1, \ldots, N} \Lambda_{i, 1}(N \lambda)-|\log r|\right\}\right\} .
\end{aligned}
$$

Corollary 11: Let Assumptions A, B and 3 hold, and let, in addition, $|\log r|>0$. Consider the family of distributed detectors in (13) and (14) with thresholds $\gamma \in\left(\bar{\gamma}_{0}, \bar{\gamma}_{1}\right)$. Then:

(a) At each sensor $i$ :

$$
\liminf _{k \rightarrow \infty}-\frac{1}{k} \log P_{\mathrm{e}, \mathrm{i}}(k, \gamma) \geq \min \left\{B_{0}(\gamma), B_{1}(\gamma)\right\}>0,
$$

and the lower bound in (58) is maximized for the point $\gamma^{\star} \in\left(\bar{\gamma}_{0}, \bar{\gamma}_{1}\right)$ at which $B_{0}\left(\gamma^{\star}\right)=B_{1}\left(\gamma^{\star}\right)$.

(b) Consider $\lambda^{\bullet}=\arg \min _{\lambda \in[0,1]} \sum_{i=1}^{N} \Lambda_{i, 0}(\lambda)$, and let:

$$
\begin{aligned}
& \operatorname{thr}\left(\Lambda_{1,0}, \ldots, \Lambda_{N, 0}\right) \\
&=\max \left\{\max _{i=1, \ldots, N} \Lambda_{i, 0}\left(N \lambda^{\bullet}\right)-\sum_{i=1}^{N} \Lambda_{i, 0}\left(\lambda^{\bullet}\right),\right. \\
&\left.\max _{i=1, \ldots, N} \Lambda_{i, 0}\left(1-N\left(1-\lambda^{\bullet}\right)\right)-\sum_{i=1}^{N} \Lambda_{i, 0}\left(\lambda^{\bullet}\right)\right\} .
\end{aligned}
$$

Then, when $|\log r| \geq \operatorname{thr}\left(\Lambda_{1,0}, \ldots, \Lambda_{N, 0}\right)$, each sensor $i$ with the detector threshold set to $\gamma=0$, is asymptotically optimal:

$$
\lim _{k \rightarrow \infty}-\frac{1}{k} \log P_{\mathrm{e}, \mathrm{i}}(k, 0)=C_{\mathrm{tot}} .
$$

Comparing Theorem 5 with Theorem 10, we can see that, under non-identically distributed observations, it is no longer possible to analytically characterize the lower bounds on the error exponents, $B_{0}(\gamma)$ and $B_{1}(\gamma)$. However, the objective functions (in the variable $\lambda$ ) in (56) and (57) are concave (by convexity of the LMGF's) and the underlying optimization variable $\lambda$ is a scalar, and, thus, $B_{0}(\gamma)$ and $B_{1}(\gamma)$ can be efficiently found by a one dimensional numerical optimization procedure, e.g., a subgradient algorithm [36].

The proof of Theorem 10 mimics the proof of Theorem 5; we focus only on the steps that account for different sensors' LMGF's. The proof of Corollary 11 is omitted due to the lack of space.

Proof of Theorem 10: First, expression (29) that upper bounds the probability of false alarm $\alpha_{i}(k, \gamma)$ for the case of non-identically distributed observations becomes:

$$
\begin{array}{r}
\mathbb{E}\left[e^{N \lambda \sum_{t=1}^{k} \sum_{j=1}^{N} \Phi_{i, j}(k, t) L_{j}(t)} \mid H_{0}\right] \\
=\mathbb{E}\left[\mathbb { E } \left[e^{N \lambda \sum_{t=1}^{k} \sum_{j=1}^{N} \Phi_{i, j}(k, t) L_{j}(t)} \mid\right.\right. \\
\left.\left.H_{0}, W(1), \ldots, W(k)\right]\right] \\
=\mathbb{E}\left[e^{\sum_{t=1}^{k} \sum_{j=1}^{N} \Lambda_{j, 0}\left(N \lambda \Phi_{i, j}(k, t)\right)}\right] .
\end{array}
$$

Next, we bound the sum in the exponent of the previous equation, conditioned on the event $\mathcal{A}_{s}$, for a fixed $s$ in $\{0,1 \ldots, k\}$, deriving a counterpart to Lemma 8 .

Lemma 12: Let Assumptions A, B, and 3 hold. Then,

(a) For any realization of $W(t), t=1,2, \ldots, k$ :

$\sum_{j=1}^{N} \Lambda_{j, 0}\left(N \lambda \Phi_{i, j}(k, t)\right) \leq \max _{j=1, \ldots, N} \Lambda_{j, 0}(N \lambda), \forall t=1, \ldots, k$.

(b) Consider a fixed $s$ in $\{0,1, \ldots, k\}$. If the event $\mathcal{A}_{s}$ occurred, then, for $i=1, \ldots, N$ :

$$
\begin{aligned}
\sum_{j=1}^{N} \Lambda_{j, 0}\left(N \lambda \Phi_{i, j}(k, t)\right) & \\
\leq \sum_{j=1}^{N} \max ( & \Lambda_{j, 0}(\lambda-\epsilon N \sqrt{N} \lambda), \\
& \left.\Lambda_{j, 0}(\lambda+\epsilon N \sqrt{N} \lambda)\right), \forall t=1, \ldots, s .
\end{aligned}
$$

The remainder of the proof proceeds analogously to the proof of Theorem 5 .

\section{CONCLUSION}

We analyzed the large deviations performance (error exponent) of consensus+innovations distributed detection over random networks. The sensors' observations have generic (non-Gaussian) distribution, independent, not necessarily identical over space, and i.i.d. in time. Our results hold assuming that the log-moment generating functions of each sensor's log-likelihood ratio are finite. We showed that the distributed detector exhibits a phase transition behavior with respect to the network connectivity, measured by $|\log r|$, where $r$ is the (exponential) rate of convergence in probability of the product $W(k) W(k-1) \cdots W(1)$ to the consensus matrix $J:=\left(\frac{1}{N}\right) 11^{\top}$. When $|\log r|$ is above the threshold, the distributed detector has the same error exponent as the optimal 
centralized detector. We further showed that the optimality threshold depends on the type of the distribution of the sensor observations. Numerical and analytical studies illustrated this dependence for Gaussian, Laplace, and binary distributions of the sensors' observations.

\section{APPENDIX}

Proof of finiteness of the log-moment generating function under (7)-(10). We now show that Assumption 2 holds, i.e., that $\Lambda_{0}(\cdot)$ is finite for any $\lambda \in \mathbb{R}$, if (7) and (9) hold. The other combinations for finiteness of $\Lambda_{0}(\cdot)$ when 1) either (7) or (8); and 2) either (9) or (10) hold can be shown similarly, and, hence, for brevity, we do not consider these cases. Assume $m>0$ (the case $m<0$ can be treated analogously), fix $\lambda \in \mathbb{R}$ and consider:

$$
\Lambda_{0}(\lambda)=\log \int_{y=-\infty}^{+\infty} e^{\lambda \log \frac{f_{n}(y-m)}{f_{n}(y)}} f_{n}(y) d y
$$

where we use the fact that the density under $H_{1}$ is $f_{1}(y)=$ $f_{n}(y-m)$, i.e., $f_{1}(\cdot)$ is the shifted density $f_{n}(\cdot)$ (of the noise) under $H_{0}$. With $f_{n}(y)=c e^{-g(y)},(60)$ is rewritten as:

$$
\begin{aligned}
e^{\Lambda_{0}(\lambda)}= & c \int_{y=-\infty}^{+\infty} e^{\lambda[-g(y-m)+g(y)]} e^{-g(y)} d y \\
= & c \int_{y=-\infty}^{+\infty} e^{-g(y)\left[1-\lambda\left(1-\frac{g(y-m)}{g(y)}\right)\right]} d y \\
= & c \int_{y=-\infty}^{0} e^{-g(y)\left[1-\lambda\left(1-\frac{g(y-m)}{g(y)}\right)\right]} d y \\
& +c \int_{y=0}^{+\infty} e^{-g(y)\left[1-\lambda\left(1-\frac{g(y-m)}{g(y)}\right)\right]} d y .
\end{aligned}
$$

Now, by (7), for any $\epsilon_{1} \in(0, \infty)$, there exists $M_{1} \in(0, \infty)$, so that

$$
\left(\left(\rho_{+}\right)-\epsilon_{1}\right) y^{\tau_{+}} \leq g(y) \leq\left(\left(\rho_{+}\right)+\epsilon_{1}\right) y^{\tau_{+}}, \forall y \geq M_{1} .
$$

Further, we have that:

$$
\begin{aligned}
&\left(\left(\rho_{+}\right)-\epsilon_{1}\right)(y-m)^{\tau_{+}} \leq g(y-m) \\
& \leq\left(\left(\rho_{+}\right)+\epsilon_{1}\right)(y-m)^{\tau_{+}}, \forall y \geq M_{1}+m .
\end{aligned}
$$

Also, for any $\epsilon_{2} \in(0, \infty)$, there exists $M_{2} \in(0, \infty)$, such that:

$$
\begin{aligned}
\left(1-\epsilon_{2}\right)(y-m)^{\tau_{+}} & \leq y^{\tau_{+}} \\
& \leq\left(1+\epsilon_{2}\right)(y-m)^{\tau_{+}}, \forall y \geq M_{2} .
\end{aligned}
$$

Now, combining (61) and (62), we obtain:

$$
\begin{aligned}
\left(1-\epsilon_{2}\right) \frac{\left(\rho_{+}\right)-\epsilon_{1}}{\left(\rho_{+}\right)+\epsilon_{1}} \\
\leq \frac{g(y-m)}{g(y)} \\
\leq\left(1+\epsilon_{2}\right) \frac{\left(\rho_{+}\right)+\epsilon_{1}}{\left(\rho_{+}\right)-\epsilon_{1}}, \\
\quad \forall y \geq M_{3}:=\max \left\{M_{1}+m, M_{2}\right\} .
\end{aligned}
$$

To upper bound the integral $\int_{y=0}^{+\infty} e^{-g(y)\left[1-\lambda\left(1-\frac{g(y-m)}{g(y)}\right)\right]} d y$, we note that, by (63), we can choose $M_{3}$ large enough, so that: $\left|1-\frac{g(y-m)}{g(y)}\right| \leq \frac{\epsilon_{3}}{|\lambda|}, \forall y \geq M_{3}$, for arbitrary $\epsilon_{3} \in(0,1)$. Thus, we have:

$$
\begin{aligned}
\int_{y=0}^{+\infty} e^{-g(y)\left[1-\lambda\left(1-\frac{g(y-m)}{g(y)}\right)\right]} d y \\
=\int_{y=0}^{M_{3}} e^{-g(y)\left[1-\lambda\left(1-\frac{g(y-m)}{g(y)}\right)\right]} d y \\
\quad+\int_{y=M_{3}}^{+\infty} e^{-g(y)\left[1-\lambda\left(1-\frac{g(y-m)}{g(y)}\right)\right]} d y \\
\leq M_{4}+\int_{y=M_{3}}^{+\infty} e^{-g(y)\left[1-|\lambda| \frac{\epsilon_{3}}{|\lambda|}\right]} d y \\
\leq M_{4}+\int_{y=M_{3}}^{+\infty} e^{-\left(1-\epsilon_{3}\right) g(y)} d y \\
\leq M_{4}+M_{5}<\infty .
\end{aligned}
$$

Finiteness of the integral $\int_{y=-\infty}^{0} e^{-g(y)\left[1-\lambda\left(1-\frac{g(y-m)}{g(y)}\right)\right]} d y$, using (9), can be proved in an analogous way. As $\lambda \in \mathbb{R}$ is arbitrary, we conclude that $\Lambda_{0}(\lambda)<+\infty, \forall \lambda \in \mathbb{R}$.

\section{REFERENCES}

[1] D. Bajović, D. Jakovetić, J. Xavier, B. Sinopoli, and J. M. F. Moura, "Distributed detection via Gaussian running consensus: Large deviations asymptotic analysis," IEEE Trans. Signal Process., vol. 59, no. 9, pp. 4381-4396, Sep. 2011.

[2] R. Viswanatan and P. R. Varshney, "Decentralized detection with multiple sensors: Part I-fundamentals," Proc. IEEE, vol. 85, no. 1, pp. 54-63, Jan. 1997.

[3] R. S. Blum, S. A. Kassam, and H. V. Poor, "Decentralized detection with multiple sensors: Part II-advanced topics," Proc. IEEE, vol. 85, pp. 64-79, Jan. 1997.

[4] J. F. Chamberland and V. V. Veeravalli, "Wireless sensors in distributed detection applications," IEEE Signal Process. Mag., vol. 24, no. 3, pp. 16-25, May 2007.

[5] J. F. Chamberland and V. Veeravalli, "Decentralized dectection in sensor networks," IEEE Trans. Signal Process., vol. 51, no. 2, pp. 407-416, Feb. 2003.

[6] J. F. Chamberland and V. Veeravalli, "Asymptotic results for decentralized detection in power constrained wireless sensor networks," IEEE J. Sel. Areas Commun., vol. 22, no. 6, pp. 1007-1015, Aug. 2004

[7] S. A. Aldosari and J. M. F. Moura, "Detection in sensor networks: The saddlepoint approximation," IEEE Trans. Signal Process., vol. 55, no. 1, pp. 327-340, Jan. 2007.

[8] D. Bajović, B. Sinopoli, and J. Xavier, "Sensor selection for event detection in wireless sensor networks," IEEE Trans. Signal Process., vol. 59, no. 10, pp. 4938-4953, Oct. 2011.

[9] J. Fang, H. Li, Z. Chen, and S. Li, "Optimal precoding design and power allocation for decentralized detection of deterministic signals," IEEE Trans. Signal Process., vol. 60, no. 6, pp. 3149-3163, Jun. 2012.

[10] S. Kar, S. A. Aldosari, and J. M. F. Moura, "Topology for distributed inference on graphs," IEEE Trans. Signal Process., vol. 56, no. 6, pp. 2609-2613, Jun. 2008.

[11] M. Alanyali, S. Venkatesh, and O. Savas, "Distributed detection in sensor networks with packet losses and finite capacity links," IEEE Trans. Signal Process., vol. 54, no. 11, pp. 4118-4132, Nov. 2006.

[12] S. A. Aldosari and J. M. F. Moura, "Topology of sensor networks in distributed detection," in ICASSP'06, IEEE Int. Conf. Acoust., Speech Signal Process., Toulouse, France, May 2006, vol. 5, pp. 1061-1064. 
[13] S. Kar, S. Aldosari, and J. M. F. Moura, "Topology for distributed inference on graphs," IEEE Trans. Signal Process., vol. 56, no. 6, pp. 2609-2613, Jun. 2008.

[14] C. G. Lopes and A. H. Sayed, "Diffusion least-mean squares over adaptive networks: Formulation and performance analysis," IEEE Trans. Signal Process., vol. 56, no. 7, pp. 3122-3136, Jul. 2008.

[15] S. Kar, J. M. F. Moura, and K. Ramanan, "Distributed parameter estimation in sensor networks: Nonlinear observation models and imperfect communication," IEEE Trans. Inf. Theory, vol. 58, no. 6, pp. 3575-3605, Jun. 2012.

[16] I. D. Schizas, G. Mateos, and G. B. Giannakis, "Distributed LMS for consensus-based in-network adaptive processing," IEEE Trans. Signal Process., vol. 57, no. 6, pp. 2365-2381, Jun. 2009.

[17] G. Mateos, I. D. Schizas, and G. B. Giannakis, "Distributed recursive least-squares for consensus-based in-network adaptive estimation," IEEE Trans. Signal Process., vol. 57, no. 11, pp. 4583-4588, Nov. 2009.

[18] F. S. Cattivelli and A. H. Sayed, "Diffusion LMS strategies for distributed estimation," IEEE Trans. Signal Process., vol. 58, no. 3, pp. 1035-1048, Mar. 2010.

[19] F. S. Cattivelli and A. H. Sayed, "Distributed detection over adaptive networks based on diffusion estimation schemes," in Proc. IEEE SPAWC '09, 10th IEEE Int. Workshop on Signal Process. Adv. in Wireless Commun., Perugia, Italy, Jun. 2009, pp. 61-65.

[20] F. S. Cattivelli and A. H. Sayed, "Diffusion LMS-based detection over adaptive networks," in Proc. Asilomar Conf. Signals, Syst. Comput., Pacific Grove, CA, Oct. 2009, pp. 171-175.

[21] P. Braca, S. Marano, V. Matta, and P. Willet, "Asymptotic optimality of running consensus in testing binary hypothesis," IEEE Trans. Signal Process., vol. 58, no. 2, pp. 814-825, Feb. 2010.

[22] F. Cattivelli and A. Sayed, "Distributed detection over adaptive networks using diffusion adaptation," IEEE Trans. Signal Process., vol. 59, no. 5, pp. 1917-1932, May 2011.

[23] S. Kar, R. Tandon, H. V. Poor, and S. Cui, "Distributed detection in noisy sensor networks," in Proc. ISIT 2011, Int. Symp. Inf. Theory, Saint Petersburg, Russia, Aug. 2011, pp. 2856-2860.

[24] S. S. Stankovic, N. Ilic, M. S. Stankovic, and K. H. Johansson, "Distributed change detection based on a consensus algorithm," IEEE Trans. Signal Process., vol. 59, no. 12, pp. 5686-5697, Dec. 2011.

[25] S. S. Stankovic, N. Ilic, M. S. Stankovic, and K. H. Johansson, "Distributed change detection based on a randomized consensus algorithm," in Proc. ECCSC '10, 5th Eur. Conf. Circuits and Syst. Commun., Belgrade, Serbia, Mar. 2010, pp. 51-54.

[26] S. A. Kassam, Signal Detection in Non-Gaussian Noise. New York: Springer-Verlag, 1987.

[27] D. Bajović, D. Jakovetić, J. Xavier, B. Sinopoli, and J. M. F. Moura, "Distributed detection over time varying networks: Large deviations analysis," in Proc. 48th Allerton Conf. Commun., Contr., Comput., Monticello, IL, Oct. 2010, pp. 302-309.

[28] D. Jakovetić, J. M. F. Moura, and J. Xavier, "Distributed detection over noisy networks: Large deviations analysis," IEEE Trans. Signal Process., vol. 60, no. 8, pp. 4306-4320, Aug. 2012.

[29] F. d. Hollander, Large Deviations. New York: Fields Inst. Monographs, American Math. Soc., 2000.

[30] M. J. Wainwright and M. I. Jordan, "Graphical models, exponential families, and variational inference," Found. Trends in Mach. Learn., vol. 1, no. 1-305, pp. 4938-4953, Oct. 2008.

[31] A. Dembo and O. Zeitouni, Large Deviations Techniques and Applications. Boston, MA: Jones and Barlett, 1993.

[32] D. Bajović, J. Xavier, J. M. F. Moura, and B. Sinopoli, "Consensus and products of random stochastic matrices: exact rate for convergence in probability," Feb. 2012 [Online]. Available: http://arxiv.org/abs/1202. 6389, submitted for publication

[33] A. F. Karr, Probability. New York: Springer-Verlag, 1993.

[34] S. Boyd and L. Vandenberghe, Convex Optimization. Cambridge, U.K.: Cambridge Univ. Press, 2004.

[35] S. Kar and J. M. F. Moura, "Distributed average consensus in sensor networks with random link failures," in ICASSP '07, IEEE Int. Conf. on Acoust., Speech Signal Process., Pacific Grove, CA, Apr. 2007, vol. 2, pp. II-1013-II-1016.

[36] J.-B. H. U. Urruty and C. Lemarechal, Convex Analysis and Minimization Algorithms: Part 1: Fundamentals, ser. Grundlehren der Mathematischen Wissenschaften 305, 306. Berlin, Germany: Springer-Verlag, 1993.



Dragana Bajović (S'11) received the Engineer Diploma from the School of Electrical Engineering, Department of Automatic Control, University of Belgrade, in 2007.

She is currently working toward the Ph.D. degree in the Dual Ph.D. program between Carnegie-Mellon University, Pittsburgh, PA, and the Instituto de Sistemas e Robótica (ISR), Instituto Superior Técnico (IST), Lisbon, Portugal. Her research interests are in the area of optimization, dimensionality reduction, and information processing in sensor networks.

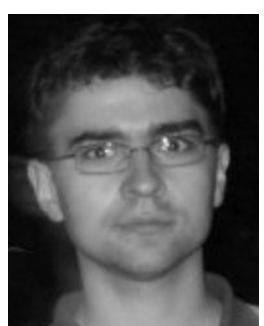

Dušan Jakovetić (S'10) received the dipl. ing. diploma from the School of Electrical Engineering, University of Belgrade, in 2007.

Currently, he is working toward the Ph.D. degree in electrical and computer engineering within the joint program of Carnegie Mellon University, Pittsburgh, PA, and the Instituto de Sistemas e Robótica (ISR), Instituto Superior Técnico (IST), Lisbon, Portugal. His research interests include consensus, distributed inference, and distributed optimization.

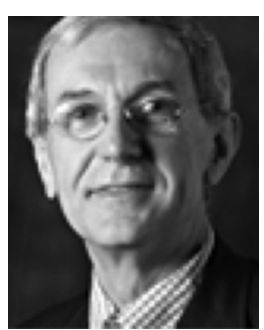

José M. F. Moura (S'71-M'75-SM'90-F'94) received the engenheiro electrotécnico degree from Instituto Superior Técnico (IST), Lisbon, Portugal, and the M.Sc., E.E., and D.Sc. degrees in electrical engineering and computer science from the Massachusetts Institute of Technology (MIT), Cambridge.

$\mathrm{He}$ is a University Professor at Carnegie Mellon University (CMU), Pittsburgh, PA, with the Departments of Electrical and Computer Engineering and, by courtesy, BioMedical Engineering. He was on the faculty at IST, has held visiting faculty appointments at MIT, and was a Visiting Research Scholar at the University of Southern California, Los Angeles. He is a founding co-director of the Center for Sensed Critical Infrastructures Research (CenSCIR) and manages a large education and research program between CMU and Portugal, www.cmuportugal.org. His research interests include statistical and algebraic signal processing, image, bioimaging, and video processing and digital communications. He has published more than 450 technical Journal and Conference papers, filed 11 patents with eight issued by the U.S. Patent Office. He is the co-editor of two books, and has given numerous invited seminars at international conferences, US and European Universities, and industrial and government Laboratories.

Dr. Moura is the Director (2013) of IEEE Division IX and currently serves on the IEEE Publications Services and Products Board. He was the President (2008-2009) of the IEEE Signal Processing Society (SPS), Editor- in-Chief for the IEEE TRANSACtions IN Signal Processing, interim Editor-in-Chief for the IEEE SignAL Processing LETTERS, and was on the Editorial Board of several journals, including the IEEE ProceEdings, the IEEE Signal Processing Magazine, and the ACM Transactions on Sensor Networks. He has been on the steering and technical committees of several Conferences. He is a Fellow of the American Association for the Advancement of Science (AAAS), and a corresponding member of the Academy of Sciences of Portugal (Section of Sciences). He was awarded the 2003 IEEE Signal Processing Society Meritorious Service Award and in 2000 the IEEE Millennium Medal. In 2007, he received the CMU's College of Engineering Outstanding Research Award and in 2009 the 2008, Philip L. Dowd Fellowship Award for Contributions to Engineering Education. In 2010, he was elected University Professor at CMU and received the IEEE Signal Processing Technical Achievement Award. He is affiliated with several IEEE societies, Sigma Xi, AAAS, AMS, IMS, and SIAM. 




João Xavier (S'97-M'03) received the Ph.D. degree in electrical and computer engineering from the Instituto Superior Técnico (IST), Technical University of Lisbon, Lisbon, Portugal, in 2002.

Currently, he is an Assistant Professor with the Department of Electrical and Computer Engineering, IST. He is also a Researcher with the Institute of Systems and Robotics (ISR), Lisbonl. His current research interests are in the area of optimization and statistical inference for distributed systems.

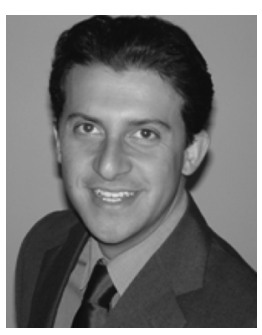

Bruno Sinopoli (M’03) received the Dr. Eng. degree from the University of Padova in 1998 and the M.S and $\mathrm{Ph} . \mathrm{D}$. degrees in electrical engineering from the University of California (UC) at Berkeley, in 2003 and 2005 respectively.

After a postdoctoral position at Stanford University, Stanford, CA, he joined the faculty at Carnegie-Mellon University, Pittsburgh, PA, where he is an Associate Professor with the Department of Electrical and Computer Engineering with courtesy appointments in Mechanical Engineering and in the Robotics Institute. His research interests include networked embedded control systems, distributed estimation and control over wireless sensor-actuator networks, and cyberphysical systems security.

Dr. Sinopoli was awarded the 2006 Eli Jury Award for Outstanding Research Achievement in the areas of systems, communications, control and signal processing at UC Berkeley, the NSF Career award in 2010, and the George Tallman Ladd Research Award by Carnegie Mellon in 2010. 